Check for updates

Cite this: Phys. Chem. Chem. Phys., 2019, 21, 4394

Received 29th October 2018, Accepted 22nd January 2019

DOI: $10.1039 / c 8 c p 06719 h$

rsc.li/pccp

\section{Magnetic-field-tuned phase transition of a molecular material from the isolated-spin to the coupled-spin regime $\dagger$}

\author{
Vinicius T. Santana, (D)*ab Beatriz N. Cunha, (D) ${ }^{c d}$ Ana M. Plutín, \\ Rafael G. Silveira, (iD cd Eduardo E. Castellano, ${ }^{b}$ Alzir A. Batista, (iD ${ }^{c}$ Rafael Calvo (iD ${ }^{f}$ \\ and Otaciro R. Nascimento (D) *b
}

\begin{abstract}
We report the preparation, X-ray structure, chemical properties, and electron paramagnetic resonance (EPR) studies at $Q$ and $X$-bands and temperature (mainly) $T=293 \mathrm{~K}$ of powder and oriented single crystal samples of the new compound [Cu( $N^{\prime}, N^{\prime}$-dimethyl- $N^{\prime}$-benzoylthiourea) $\left(2,2^{\prime}\right.$-bipyridine $\left.) C l\right]$, called CuBMB. The EPR spectra of single crystal samples at the $Q$-band display abrupt merging and narrowing of the peaks corresponding to two rotated copper sites as a function of magnetic field $\left(\boldsymbol{B}_{0}\right)$ orientation. This behaviour indicates a quantum transition from an array of quasi-isolated spins to a quantumentangled spin array associated with exchange narrowing processes and produced by weak intermolecular exchange interactions $J_{i}$ between neighbour copper spins. This transition occurs when the magnitudes of the anisotropic contributions to the Zeeman couplings, tuned with the direction of $\boldsymbol{B}_{0}$, approach these $\left|J_{i}\right|$ and produce level crossings. The exchange couplings between neighbour spins are estimated from the angular variation of the single crystal EPR results at the Q-band. We analyse the quantum behaviour and phase transitions of the spin system and discuss the magnitudes of the exchange couplings in terms of the structure of the chemical paths connecting Cu neighbours. The single crystal data at the Q-band indicates an uncommon ground electronic state of $\mathrm{Cu}^{\text {Il }}$ which is discussed and compared with the results of DFT calculations. The spectrum of polycrystalline (powder) samples at the Q-band is a sum of contributions of microcrystals in each phase, and the fraction $F$ of the entangled phase depends on the microwave frequency. The X-band spectrum is compatible with the $Q$-band results, but does not display a transition, and the spin system is in the quantum-entangled phase for all field orientations. This behaviour is further studied with a simple geometric model giving basic predictions. The crystal structure of CuBMB is monoclinic, space group $P 2_{1} / n$, with $a=11.9790(3) \AA, b=14.0236(5) \AA, c=12.1193(3) \AA, \beta=104.952(2)^{\circ}$ and $Z=4$, and the copper ions are equatorially bonded to the benzoylthiourea and bipyridine ligands in a heavily distorted square pyramidal structure.
\end{abstract}

${ }^{a}$ CEITEC - Central European Institute of Technology, Brno University of Technology, Purkyňova 123, 61200 Brno, Czech Republic. E-mail: vinicius.santana@ceitec.vutbr.cz; Tel: 420541149265

${ }^{b}$ Departamento de Física e Ciencias Interdisciplinares, Instituto de Física de São Carlos, Universidade de São Paulo - USP, CP 369, 13560-970, São Carlos, SP, Brazil. E-mail: ciro@ifsc.usp.br

${ }^{c}$ Departamento de Quimica, Universidade Federal de São Carlos - UFSCar, Rodovia Washington Luis Km 235, CP 676, 13565-905, São Carlos, SP, Brazil

${ }^{d}$ Instituto Federal Goiano, Campus Ceres, Rodovia GO-154, Km 03, CP 51, 76300-000, Ceres, GO, Brazil

${ }^{e}$ Laboratorio de Sintesis Orgánica, Facultad de Quimica, Universidad de la Habana UH, Habana 10400, Cuba

${ }^{f}$ Instituto de Física del Litoral, CONICET-UNL, and Departamento de Física, Facultad de Bioquimica y Ciencias Biológicas, Universidad Nacional del Litoral, Güemes 3450, 3000 Santa Fe, Argentina

$\dagger$ Electronic supplementary information (ESI) available. CCDC 1866815. For ESI and crystallographic data in CIF or other electronic format see DOI: 10.1039/c8cp06719h

\section{Introduction}

Molecular materials are part of a multidisciplinary research field involving physicists, chemists, biochemists and technologists aiming to prepare, to characterize, and to use materials obtained by assembling together simpler inorganic and/or organic compounds. They are useful for basic and for technical applications (electrical, magnetic, optical, etc.), ${ }^{1,2}$ and also for biological purposes (e.g., pharmacological applications ${ }^{3,4}$ ). The study of new compounds in connection with their applications promoted progress in coordination chemistry, and understanding the relationships between the molecular structure and the properties provides important tools to aid the design of new compounds to be applied for specific purposes. The number and complex nature of the interactions between molecules challenge devising 
quantitative relationships between structure and behaviour. Collecting data for convenient compounds, it may be possible to understand the roles of particular structural features in the properties of the molecule. In this way, new qualitative and quantitative correlations between a parameter and its particular effects may be found.

Within the domain of molecular materials with specific magnetic properties, single-molecule magnets ${ }^{5,6}$ provide a $1: 1$ ratio between quasi-static switchable magnetic moments and molecules in the sample. An important property is the bridges between metal ions or molecules that mediate magnetic interactions between the magnetic centres they interlink. ${ }^{7}$ By molecular-programmed synthetic routes, different ligands are used as spacers acting as the bridges that build nuclearity-controlled metal assemblies. The great variety of obtained materials instigated a lot of research effort in an attempt to model thermodynamic, biological, and quantum mechanical problems.

Following investigations that started more than a century ago, ${ }^{8}$ we prepared new Cu-acylthiourea compounds relevant for their pharmacological properties. ${ }^{9,10}$ We measured their crystal structures and collected electron paramagnetic resonance (EPR) spectra in powder and oriented single crystal samples. Here we report results for the compound $\left[\mathrm{Cu}\left(N^{\prime}, N^{\prime}\right.\right.$-dimethyl- $N^{\prime}$ benzoylthiourea)(2,2'-bipyridine)Cl] (to be called CuBMB), which proved to be very appropriate for studying quantum and magnetic behaviour of materials with weak intermolecular exchange interactions $\left(J_{i}\right)$ between metal ions. CuBMB is paramagnetic, with long non-covalent chemical paths supporting very weak interactions between neighbour copper spins. Thermodynamic variables such as susceptibility, magnetization, and specific heat depend on the energies of the levels which are related to the $J_{i}$ and contribute information only in the rarely achieved range of extremely low $T \leq\left|J_{i}\right| / k_{\mathrm{B}}$. The temperature of our experiments also precludes the possibility of thermodynamic phase transitions. On the other hand, weak interactions generate traveling spin excitations related to the spin dynamics that strongly modify the EPR spectra.

Gorter and Van Vleck ${ }^{11}$ introduced the concept of exchange narrowing to explain EPR results in metal compounds. Their idea was developed by Anderson ${ }^{12,13}$ and $\mathrm{Kubo}^{14}$ into the classic theory of exchange narrowing ${ }^{15,16}$ explaining the changes of the line widths and the merging of the EPR spectra of a spin system produced by the exchange couplings. Yokota and Koide ${ }^{17}$ applied these ideas to compounds containing magnetically non-equivalent metal sites with anisotropic $g$-matrices. In those early times, detailed EPR results were scarce because of instrumental limitations, and restrained detection of fine aspects of the process. More recently, data collected from single crystals allowed evaluating selectively weak magnitudes of $J_{i}$ which may be correlated to the electronic structure of the paths connecting metal ions. ${ }^{18-27}$ In a few cases where thermodynamic data at very low $T$ also existed, good agreement was obtained between the values of $J_{i}$ obtained by both methods. ${ }^{28,29}$

The merging phenomenon in EPR was treated by Hoffmann et $a .^{18-20}$ from the point of view of classical Bloch equations. ${ }^{30}$ They collected data on small exchange coupling between metal ions at distances up to $25 \AA$ and proposed an empirical formula $^{20}$ that allows estimation of the small exchange coupling between metal spins at long distances, complementing earlier results of Coffman and Buettner, ${ }^{31}$ and providing new ideas and useful bibliographic support.

The EPR results reported here reveal novel properties of the exchange narrowing processes that manifest as a quantum phase transition between phases characterized by split and by fully merged EPR spectra of rotated copper ions in the lattice. This condition is related to the quantum entanglement of the spins, which in our experiments is tuned with the magnitude and orientation of the applied magnetic field $\boldsymbol{B}_{0}=\mu_{0} \boldsymbol{H}\left(\mu_{0}\right.$ is the permeability of a vacuum). The collective state of the spin system changes between that for an array of uncoupled $\mathrm{Cu}$ ions, to that for a quantum-entangled spin array using the anisotropy of the $\boldsymbol{g}$-matrix of the copper ions and varying the orientation of $\boldsymbol{B}_{0}$. Following the spectral changes we cross a magnetic phase transition relevant to problems such as avoided level crossings, ${ }^{32-37}$ Bose-Einstein condensation, ${ }^{38}$ interacting metal-dimeric arrays, ${ }^{39-43}$ and quantum dynamical phase transitions, ${ }^{44,45}$ where small interactions between magnetic molecules play relevant roles, ${ }^{46}$ and EPR experiments provide useful information. CuBMB has unprecedented advantages to study experimentally these effects and update the understanding of the exchange narrowing and spectral collapse phenomena of EPR lines. Our results provide a new approach to interpret the problem and, in addition, we estimate isotropic spin-spin interactions $\left|J_{i}\right| \sim 0.03-0.1 \mathrm{~cm}^{-1}$ between coppers in CuBMB and discuss their relation with the structure of the chemical paths connecting them. Regarding the individual behaviour of CuBMB molecules, our EPR studies of single crystal samples at the Q-band show that, as a consequence of the structural distortion of the copper site, the electronic wave function displays unusual characteristics that are further investigated with DFT calculations. In a future paper we will treat the pharmacological properties of $\mathrm{CuBMB}$ and of several other similar $\mathrm{Cu}$ derivatives of acylthiourea, which are mostly related to intramolecular (instead of intermolecular) interactions.

\section{Materials and methods}

\subsection{Material preparation}

The solvents used in the reactions were purified by standard methods. IR spectra were recorded on a FT-IR Bomem-Michelson 102 spectrometer in the range $4000-250 \mathrm{~cm}^{-1}$ at $293 \mathrm{~K}$, using $\mathrm{KBr}$ pellets. Elemental analyses were carried out by the micro analytical laboratory at the Chemistry Department of the Federal University of São Carlos using an EA 1108 CHNS microanalyser (Fisons Instruments). Conductivity analyses were performed using a Micronal model B-330 connected to a Pt electrode with a cell constant equal to $0.089 \mathrm{~cm}^{-1}$, using $1.0 \mathrm{mM}$ solutions of the complex in acetone. The molar conductance measurements were carried out in dichloromethane and the result $2.25 \mathrm{~S} \mathrm{~cm}^{2} \mathrm{~mol}^{-1}$ is consistent with a neutral type of compounds. The acylthiourea ligand ( $\mathrm{L}=N^{\prime}, N^{\prime}$-dimethyl- $N^{\prime}$-benzoylthiourea) was synthesized following a previous report. ${ }^{47}$ The complex $[\mathrm{Cu}($ bipy $)(\mathrm{L}) \mathrm{Cl}]$, (bipy $=$ $2,2^{\prime}$-bipyridine) was prepared in good yield $(77 \%)$ at room $T$ 


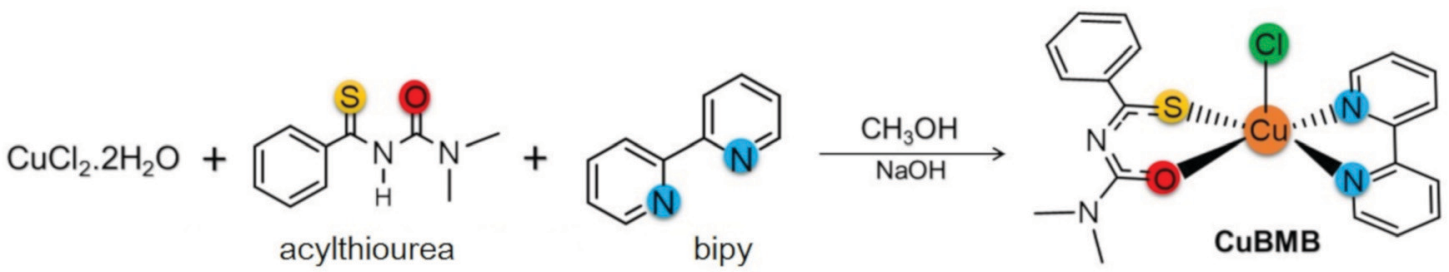

Scheme 1 Synthesis of the complex CuBMB from the ligands $L=N^{\prime}, N^{\prime}$-dimethyl- $N^{\prime}$-benzoylthiourea and 2,2'-bipyridine.

(see Scheme 1). The ligand $\mathrm{L}(0.13 \mathrm{~g}, 0.64 \mathrm{mmol})$ and $\mathrm{NaOH}$ $(0.03 \mathrm{~g}, 0.64 \mathrm{mmol})$ dissolved in $5 \mathrm{~mL}$ of methanol were added to a methanolic solution $(15 \mathrm{~mL})$ of $\mathrm{CuCl}_{2} \cdot 2 \mathrm{H}_{2} \mathrm{O}(0.10 \mathrm{~g}$, $0.59 \mathrm{mmol}$ ) and stirred for $0.5 \mathrm{~h}$. Afterwards, the bipyridine ligand $(0.10 \mathrm{~g}, 0.64 \mathrm{mmol})$, previously dissolved in methanol $(5 \mathrm{~mL})$, was added and the resulting solution was stirred for $5 \mathrm{~h}$. The product was filtered off, washed with diethyl ether $(10 \mathrm{~mL})$ and dried under a vacuum. Emerald-green color crystals were obtained by slow evaporation of dichloromethane/methanol solution. The complex is air-stable, exhibiting satisfactory elemental analysis data.

\subsection{X-ray diffractometer and software}

Single crystal X-ray diffraction measurements were performed on an Enraf-Nonius Kappa-CCD and Apex II Duo diffractometer with graphite monochromated $\operatorname{MoK} \alpha$ radiation $(\lambda=0.71073 \AA)$. Data collection was carried out using the Collect software ${ }^{48}$ and the structure was obtained by the direct method using SHELXS-97. ${ }^{49}$ The Gaussian absorption correction was applied, ${ }^{50}$ and tables and structure representations were generated by WinGX ${ }^{51}$ and Mercury, ${ }^{52}$ respectively.

\subsection{EPR methods}

We used a Varian EPR spectrometer working at $\sim 35.2 \mathrm{GHz}$ (Q-band) and at $\sim 9.52 \mathrm{GHz}$ (X-band). A single crystal of $\sim 2 \times$ $1.5 \times 1 \mathrm{~mm}^{3}$ was mounted with the $a c$ plane glued with vacuum grease on top of a cubic sample holder made of cleaved $\mathrm{KCl}$, as displayed in Fig. 1 (sample montage $\boldsymbol{a} \boldsymbol{b} \boldsymbol{c}^{*}$ ). After collecting the EPR data for this sample, the crystal was rotated $40^{\circ}$ as shown in Fig. $1 \mathrm{~b}$ (sample montage $\boldsymbol{v b u}$ ), so the $a c^{*}$ plane in sample $\boldsymbol{a} \boldsymbol{b} \boldsymbol{c}^{*}$, and the $u v$ plane in sample $\boldsymbol{v} \boldsymbol{b u}$, differ by a shift of $40^{\circ}$ in the angular coordinate. In each case, the sample-holder was glued with different faces in the horizontal plane on top of a pedestal inside the microwave cavity in order to collect spectra with $\boldsymbol{B}_{0}$ in the planes parallel to the faces of the holder, rotating the magnet (Q-band), or the holder with a goniometer (X-band). Most measurements were performed at $T=293 \mathrm{~K}$, with $\sim 0.1 \mathrm{mT}$ amplitude of $100 \mathrm{kHz}$ field modulation, and $10 \mathrm{~mW}$ microwave power, precluding spectral saturation. We calibrated $\boldsymbol{B}_{0} / \nu$ measuring the position of the signal of a paramagnetic $\mathrm{Cr}^{\mathrm{III}}: \mathrm{MgO}$ spin marker next to the sample $(g=1.9797)$. Powder spectra are labeled by the microwave frequency. Single crystals also require for each spectrum three Euler angles of a laboratory frame $X Y Z$,

‡ Elemental analysis (\%) for $\mathrm{C}_{20} \mathrm{H}_{19} \mathrm{ClCuN}_{4} \mathrm{OS}$ : exp. (calc.) C, 51.95 (51.94); H, 4.31 (4.14); N, 12.15 (12.12); S 7.09 (6.93). (a) $\boldsymbol{a b c} *$ sample

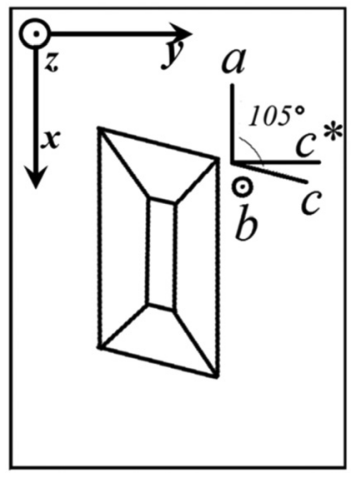

Fig. 1 Orientations of the single crystal sample in the holder: (a) Sample $\boldsymbol{a} \boldsymbol{b} \boldsymbol{c}^{\star}\left(\boldsymbol{c}^{\star}=\boldsymbol{a} \times \boldsymbol{b}\right)$; (b) sample $\boldsymbol{v b u}$. Sample $\boldsymbol{a} \boldsymbol{b} \boldsymbol{c}^{\star}$ allows collecting spectra with $\boldsymbol{B}_{0}$ in the $b a, b c^{\star}$ and $a c^{\star}$ planes. Sample $\boldsymbol{v} \boldsymbol{b} \boldsymbol{u}$ allows collecting spectra in the $b u, b v$ and $u v$ planes. The planes $u v$ and $a c^{*}$ are the same, except for a shift of $40^{\circ}$ in the angular coordinate. The laboratory axes $\boldsymbol{x}, \boldsymbol{y}$, and $\boldsymbol{z}$ are used as a reference for the data for each sample.

in which $\boldsymbol{B}_{0}$ is along the $\boldsymbol{Z}$ axis, the microwave field $\boldsymbol{B}_{1}$ (normal to the rotation plane of $\boldsymbol{B}_{0}$ ) is along the $\boldsymbol{X}$ axis, and $\boldsymbol{Y}=\boldsymbol{Z} \times \boldsymbol{X}$, expressed in the $\boldsymbol{x y z} \equiv \boldsymbol{a} \boldsymbol{b} \boldsymbol{c}^{*}$ or $\boldsymbol{x y z} \equiv \boldsymbol{v} \boldsymbol{b u}$ "sample" orthogonal frames (see Fig. 1a and b). These angles are needed to analyze the data because the orientation $\boldsymbol{X}$ of $\boldsymbol{B}_{1}$ determines the line intensities of single crystals. The central field $\boldsymbol{B}_{0}$ and the full width at half height (FWHM) $\Omega$ of the resonances were obtained from least squares fits of Lorentzian derivative line shapes to the observed single crystal spectra. We evaluated the $g$-values of the peaks of each spectrum from the distances in magnetic field to a field marker, whose $g$-value is well known. These distances, obtained from fits of spectra having a very high signal-to-noise ratio, are in general accurate within $0.1 \mathrm{mT}$, and better in the axes displaying minimum widths. Thus, due to the anisotropy of the spectra, the field orientation (or sample orientation) is the main source of the $g$-uncertainties in the values proposed in the single crystal measurements. We used EasySpin (v. 5.15), ${ }^{53}$ a package of programs working under Matlab, ${ }^{54}$ which together allow simulating and fitting a given Hamiltonian to spectral line shapes $\mathrm{d} \chi^{\prime \prime} / \mathrm{d} B_{0} v s$. $\boldsymbol{B}_{0}$ from powder and single crystal samples.

\subsection{DFT calculations}

For the DFT calculations we used the software Orca version 4.0.1, ${ }^{55}$ with the B3LYP hybrid functional, 6-31G basis set, and spin unrestricted SCF (UKS). Hybrid functionals have been proved to provide good performance for $\mathrm{d}^{9}$ complexes. ${ }^{56}$ The calculated 
exchange interaction $J^{\mathrm{c}}$ was obtained via the DFT-"broken symmetry" (DFT-BS) approach following the methodology discussed by Levchenkov et al. for intermolecular exchange via $\mathrm{N}-\mathrm{H} \cdots \mathrm{O}$ bonds in copper complexes. ${ }^{57}$ The coupling constant was obtained with a spin-Hamiltonian analysis based on $\mathcal{H}=$ $-2 J^{\mathrm{c}} S_{\mathrm{A}} \cdot S_{\mathrm{B}}$ and $J^{\mathrm{c}}=-\left(E_{\mathrm{HS}}-E_{\mathrm{BS}}\right) /\left[S_{\max }\left(S_{\max }+1\right)\right] .{ }^{58}$ The input files are included with the ESI. $\dagger$

\section{Experimental results}

\subsection{Crystal structure}

Crystallographic data and selected bond distances and angles for CuBMB are listed in Tables 1 and 2. The compound crystallizes in the monoclinic space group $P 2_{1} / n$ with $a=11.9790(3) \AA$, $b=14.0236(5) \AA, c=12.1193(3) \AA$ and $\beta=104.952(2)^{\circ}$, with $Z=4$. The copper ions are in five-coordinated sites (Fig. 2) and, according to the value 0.49 calculated for the parameter $\tau=$ $(\beta-\alpha) / 60$ ( $\beta$ and $\alpha$ are the two largest basal angles of a five coordinate metal, with $\tau=0$ for ideal square pyramidal coordination and $\tau=1$ for ideal trigonal pyramidal ${ }^{59}$ ), in a heavily distorted square pyramidal structure, with the chlorine atom in an axial position, and the chelated ligands bipyridine and acylthiourea (Scheme 1) in equatorial positions of the coordination sphere (Fig. 2). From this structural point of view, acylthiourea acts as an anionic ligand with bidentate equatorial coordination $(\mathrm{S}, \mathrm{O})$ and bipyridine through the equatorial nitrogens N3 and N4 to the $\mathrm{Cu}^{\mathrm{II}}$ ion. This view will be somewhat changed later by our results on the electronic structure. Upon metal coordination, the average bond lengths of thiocarbonyl and carbonyl moieties are longer than those for the free acylthiourea ligands. ${ }^{60,61}$

Table 1 Crystal data and structure refinement parameters for CuBMB

\begin{tabular}{|c|c|}
\hline Empirical formula & {$\left[\mathrm{CuC}_{20} \mathrm{H}_{19} \mathrm{~N}_{4} \mathrm{OSCl}\right]$} \\
\hline Formula weight & 462.44 \\
\hline Crystal system & Monoclinic \\
\hline Space group & $P 2_{1} / n$ \\
\hline \multicolumn{2}{|l|}{ Unit cell dimensions } \\
\hline$a(\AA)$ & $11.9790(3)$ \\
\hline$b(\AA)$ & $14.0236(5)$ \\
\hline$c(\AA)$ & $12.1193(3)$ \\
\hline$\alpha\left({ }^{\circ}\right)$ & 90 \\
\hline$\beta\left(^{\circ}\right)$ & $104.952(2)$ \\
\hline$\gamma\left({ }^{\circ}\right)$ & 90 \\
\hline Volume $\left(\AA^{3}\right)$ & $1966.97(10)$ \\
\hline$Z$ & 4 \\
\hline Density calculated $\left(\mathrm{Mg} \mathrm{m}^{-3}\right)$ & 1.562 \\
\hline$\mu\left(\mathrm{mm}^{-1}\right)$ & 1.371 \\
\hline$F(000)$ & 948 \\
\hline Crystal size $\left(\mathrm{mm}^{3}\right)$ & 0.3250 .3100 .260 \\
\hline$\theta$-range $\left(^{\circ}\right)$ & 2.579 to 25.999 \\
\hline Index ranges & $\begin{array}{l}-12 \leq h \leq 14,-15 \leq k \leq 17 \\
-14 \leq l \leq 14\end{array}$ \\
\hline Reflections collected & 16840 \\
\hline Independent reflections & $3856[R($ int $)=0.0931]$ \\
\hline Completeness to $\theta(\%)$ & 99.9 \\
\hline Refinement method & Full-matrix least-squares on $F^{2}$ \\
\hline Data/restraints/parameters & $3856 / 0 / 256$ \\
\hline Goodness-of-fit on $F^{2}$ & 1.104 \\
\hline Final $R$ indices $[I>2 \operatorname{sigma}(I)]$ & $R_{1}=0.0656, \mathrm{w} R_{2}=0.1888$ \\
\hline$R$ indices (all data) & $R_{1}=0.0791, \mathrm{w} R_{2}=0.1973$ \\
\hline$\Delta \rho_{\max }$ and $\Delta \rho_{\min }\left(\mathrm{e} \AA^{-3}\right)$ & 1.023 and -0.657 \\
\hline
\end{tabular}

Table 2 Selected interatomic distances $[\AA]$ and bond angles $\left[{ }^{\circ}\right]$ for CuBMB Bond lengths $[\AA]$

\begin{tabular}{ll}
\hline $\mathrm{Cu}-\mathrm{S} 1$ & $2.3088(15)$ \\
$\mathrm{Cu}-\mathrm{O} 1$ & $1.948(4)$ \\
$\mathrm{Cu}-\mathrm{N} 3$ & $2.081(5)$ \\
$\mathrm{Cu}-\mathrm{N} 4$ & $2.013(4)$ \\
$\mathrm{Cu}-\mathrm{Cl}$ & $2.4215(16)$ \\
$\mathrm{O} 1-\mathrm{C} 1$ & $1.264(6)$ \\
$\mathrm{S} 1-\mathrm{C} 8$ & $1.727(6)$ \\
$\mathrm{N} 1-\mathrm{C} 1$ & $1.323(7)$ \\
$\mathrm{N} 1-\mathrm{C} 8$ & $1.337(7)$ \\
$\mathrm{N} 2-\mathrm{C} 8$ & $1.339(7)$ \\
& \\
$\mathrm{Bond}$ angles $\left.{ }^{\circ}\right]$ & \\
$\mathrm{O} 1-\mathrm{Cu}-\mathrm{S} 1$ & $90.59(12)$ \\
$\mathrm{N} 3-\mathrm{Cu}-\mathrm{N} 4$ & $79.38(18)$ \\
$\mathrm{O} 1-\mathrm{Cu}-\mathrm{S} 1$ & $90.59(12)$ \\
$\mathrm{Cl}-\mathrm{Cu}-\mathrm{O} 1$ & $96.83(13)$ \\
$\mathrm{Cl}-\mathrm{Cu}-\mathrm{S} 1$ & $114.18(6)$ \\
$\mathrm{Cl}-\mathrm{Cu}-\mathrm{N} 3$ & $107.34(13)$ \\
$\mathrm{Cl}-\mathrm{Cu}-\mathrm{N} 4$ & $93.75(14)$ \\
$\mathrm{N} 3-\mathrm{Cu}-\mathrm{N} 4$ & $79.38(18)$
\end{tabular}

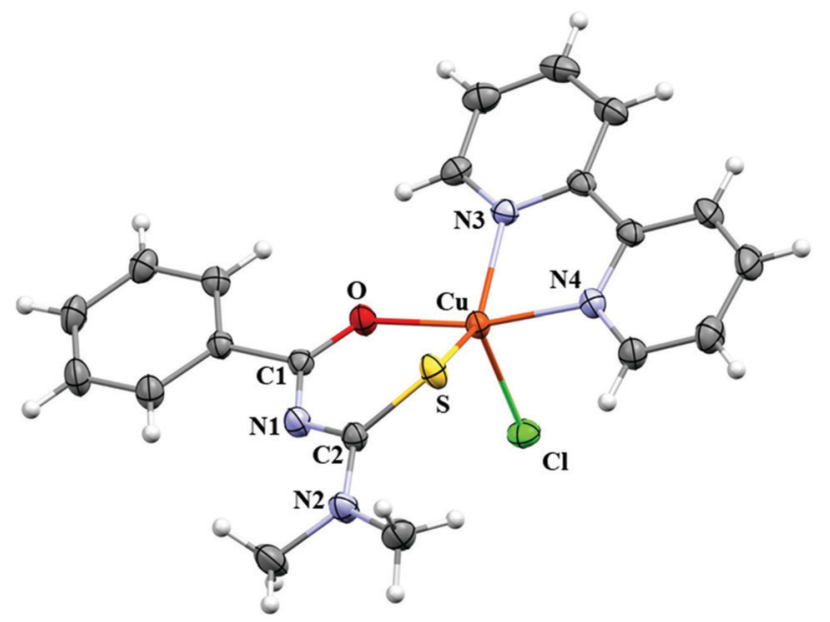

Fig. 2 Molecule of $\left[\mathrm{Cu}\left(N^{\prime}, N^{\prime}\right.\right.$-dimethyl- $N^{\prime}$-benzoylthiourea)(2,2'bipyridine) $\mathrm{Cl}$, [ $\mathrm{CuC}_{20} \mathrm{H}_{19} \mathrm{~N}_{4} \mathrm{OSCl}$, called CuBMB.

However, the $\mathrm{C}-\mathrm{N}$ distances (N1-C1/N1'-C1'; N1-C8/N1'-C8') are shorter. The changes of the bond lengths of the thiocarbonyl fragment (SCNCO) favour $\pi$-delocalization over the six-membered chelate ring, ${ }^{62,63}$ and the observed $\mathrm{Cu}-\mathrm{N}$ and $\mathrm{Cu}-\mathrm{Cl}$ bond lengths agree with reported values. ${ }^{64}$ Intermolecular interactions supporting the crystalline structure are maintained by weak hydrogen bonds $\mathrm{C}-\mathrm{H} \cdots \mathrm{O}(2.68 \AA), \mathrm{C}-\mathrm{H} \cdots \mathrm{Cl}(2.95-3.11 \AA)$, and C-H $\cdots \mathrm{N}(3.69 \AA)$, and by $\pi$ - $\pi$-stacking between the bipy and benzoyl rings with $\mathrm{C}-\mathrm{C}$ distances ranging between $3.29 \AA$ and $3.61 \AA$ (centroid-to-centroid average distances $\sim 3.56 \AA$ ). A more detailed analysis of intermolecular interactions is presented later in the context of the magnetic properties of the compound.

\subsection{Infrared bands}

The characteristic IR bands $\left(\mathrm{KBr}\right.$, in $\left.\mathrm{cm}^{-1}\right)$ of the free ligand $N^{\prime}, N^{\prime}$-dimethyl- $N^{\prime}$-benzoylthiourea are: $\nu_{\mathrm{N}-\mathrm{H}}=3200, \nu_{\mathrm{C}=\mathrm{O}}=$ $1690, \nu_{\mathrm{C}=\mathrm{S}}=1286$, and $\nu_{\mathrm{C}-\mathrm{S}}=875$. The $\mathrm{N}-\mathrm{H}$ band is absent in 
the IR of the complex, demonstrating the anionic nature of acylthiourea upon coordination to $\mathrm{Cu}$. The other bands shift to negative wavenumbers, $\nu_{\mathrm{C}=\mathrm{O}}=1595, \nu_{\mathrm{C}=\mathrm{S}}=1244$ and $\nu_{\mathrm{C}-\mathrm{S}}=784$, when compared with free ligands, indicating the $\pi$-electron delocalization induced by deprotonation of the nitrogen atom of the amide group, expected for the bidentate coordination $(\mathrm{S}, \mathrm{O})$ of acylthiourea to a metal. ${ }^{65,66}$ Weak vibrations at $\nu_{\mathrm{Cu}-\mathrm{O}}=630$, $\nu_{\mathrm{Cu}-\mathrm{N}}=500, \nu_{\mathrm{Cu}-\mathrm{S}}=412$ and $\nu_{\mathrm{Cu}-\mathrm{Cl}}=271 \mathrm{~cm}^{-1}$ in the spectrum describing stretching frequencies are observed. ${ }^{67}$

\section{3 $\quad$ EPR spectra}

EPR spectra of single crystal and polycrystalline (powder) samples were collected at the Q- and the X-band and actual values of the microwave frequency were used to calculate the $g$-factors of each spectrum. In order to improve the quality of our experimental results we found it useful to collect Q-band data in two different sets of orientations of the single crystal sample as described in the experimental section. Although it was tempting to discard the powder data after collecting such an exhaustive single crystal set of data, we found that they contain highly clarifying findings, and we included these results. No hyperfine structure of the spectra is observed for any orientation of the sample in the magnetic field.

3.3.1 Single-crystal samples. The spectra were collected at intervals of $5^{\circ}$ (or smaller in some angular ranges) over $180^{\circ}$ of each of the three main planes of samples $\boldsymbol{a} \boldsymbol{b} \boldsymbol{c}^{*}$ and $\boldsymbol{v} \boldsymbol{b} \boldsymbol{u}$ at the Q-band, and $\boldsymbol{a} \boldsymbol{b} \boldsymbol{c}^{*}$ at the X-band. At the Q-band two EPR peaks are observed for a general orientation of $\boldsymbol{B}_{0}$ (see the example in Fig. 3, collected at $293 \mathrm{~K}$ ) and they merge to one peak for $\boldsymbol{B}_{0}$ along the $\boldsymbol{b}$-axis or perpendicular to it ( $a c^{*}$ and $u v$ planes), where the coppers are magnetically equivalent. Besides, the two peaks also merge abruptly within angular ranges around these symmetry orientations. The collected spectra were fit by Lorentzian derivative line shapes $\mathrm{d} \chi^{\prime \prime} / \mathrm{d} B_{0}$ in order to calculate the line positions and peak-to-peak widths $\Omega$ of the two (or one) peaks, as a function of field orientation.

Most of the data reported in this work were collected at $293 \mathrm{~K}$ with the idea that the important information for our purpose is independent of $T$. This hypothesis was verified by collecting some

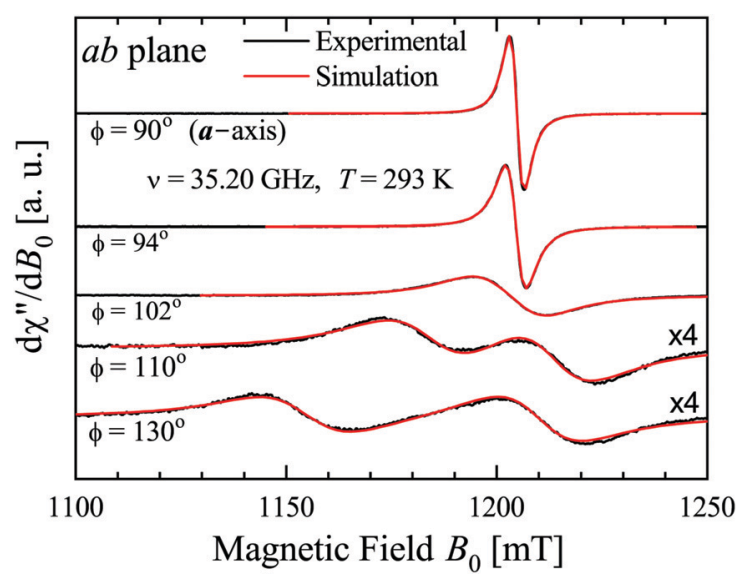

Fig. 3 EPR spectra with $\boldsymbol{B}_{0}$ in the ba plane. Two peaks merge and narrow when approaching the $\boldsymbol{a}$-axis. Experimental result (black lines), and fit (red lines) with two Lorentzian derivative line shapes are indistinguishable.

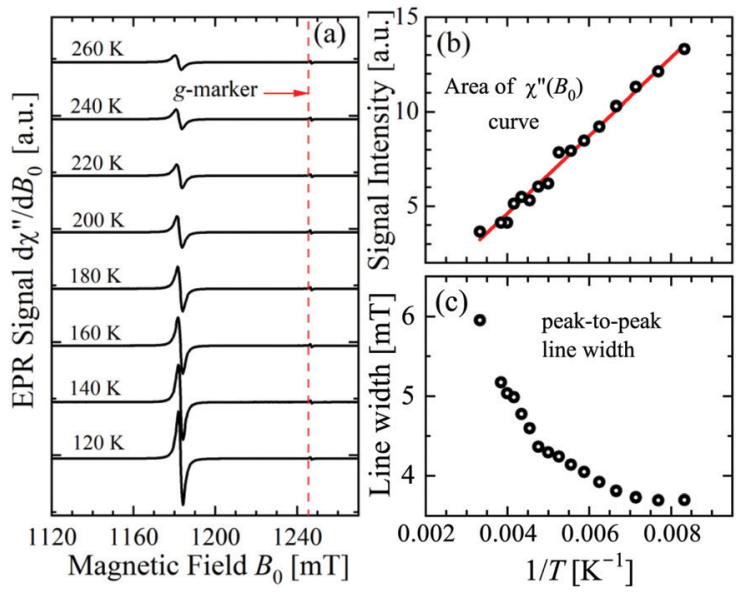

Fig. 4 Temperature dependence of the (a) EPR spectra, (b) integrated area, and (c) peak-to-peak line width measured with the magnetic field along the $\boldsymbol{a}$ axis direction in the ba plane of the single crystal.

single crystal data in the range $120 \mathrm{~K}<T<300 \mathrm{~K}$. Fig. 4a-c display spectra obtained for $\boldsymbol{B}_{0}$ along the $\boldsymbol{a}$-axis, the temperature dependences of the area of the integrated signal $\left(\chi^{\prime \prime}\left(B_{0}\right)\right)$, and the peak-to-peak width of the signal. The $1 / T$ dependence of the area clearly points to a paramagnetic behaviour. The broadening with increasing $T$ of the width of the peak may be attributed to the expected shortening of the relaxation time of the copper ions. We also measured as a function of $T$ the spectra observed in the neighborhood of the $\boldsymbol{a}$-axis, as in Fig. 3 . The broadening at these angles is the same as at the $\boldsymbol{a}$-axis, but due to the larger width of the signal, its fractional contribution to the total width is negligible.

The experimental values for the $g^{2}$-factor and the line width $\Omega$ of the peaks at the Q-band are given by symbols in Fig. 5 and 6 , where the results in all studied planes are included. They depict a merging of the resonances and a simultaneous important narrowing

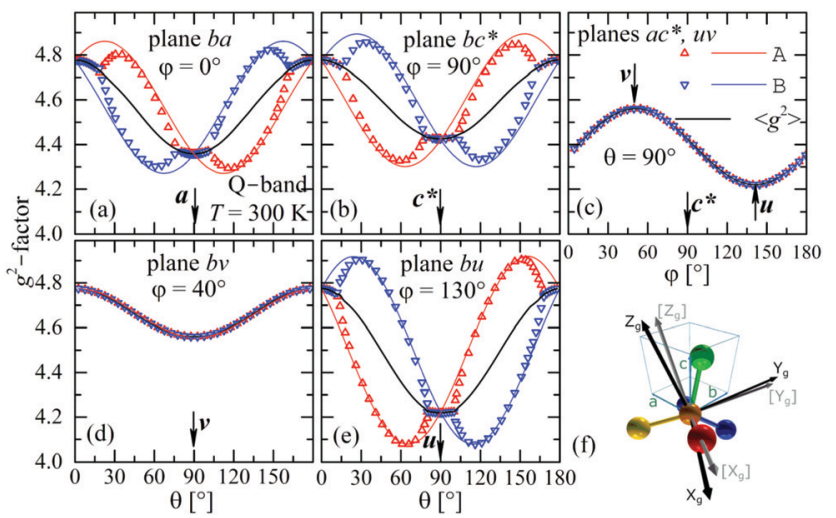

Fig. 5 Angular variation of the $g^{2}$-factor in the crystal planes ba (a), $b c^{*}$ (b) and $a c^{\star}$ (c) (sample $a b c^{\star}$ ), and bv (d) and bu (e) (sample vbu), see Fig. 1 and text. Plane $u v$ is included with $a c^{*}$. Symbols are experimental results and solid lines are obtained from global fits of eqn (3) to the data for $\boldsymbol{g}_{\mathrm{A}}{ }^{2}$ and $\boldsymbol{g}_{\mathrm{B}}{ }^{2}$ and $g^{2}$-matrices whose matrix elements are given in Table 3 . Symbols and lines with red and blue color correspond to A and B copper sites. The black lines correspond to the average $\boldsymbol{g}^{2}$-matrix (eqn (3)) and the calculated elements of $\boldsymbol{g}^{2}, \boldsymbol{g}_{\mathrm{A}}{ }^{2}$, and $\boldsymbol{g}_{\mathrm{B}}{ }^{2}$-matrices are given in Table 3 . In (f) the principal directions $\left(X_{g}, Y_{g}\right.$, $Z_{g}$ ) of the $\boldsymbol{g}$-matrix are shown together with the copper ligands in CUBMB with the $Z_{g}$ axis approximately in the plane between the $\mathrm{Cu}-\mathrm{Cl}$ and $\mathrm{Cu}-\mathrm{S}$ bonds. 


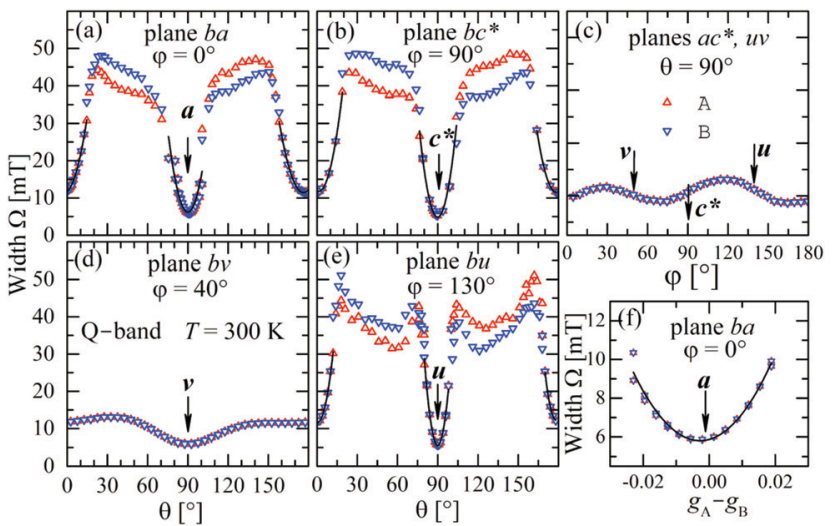

Fig. 6 Angular variation of the line width $\Omega$ for $\boldsymbol{B}_{0}$ in the crystal planes $b a, b c^{*}$ and $a c^{*}$ of sample $a b c^{*}$ and $u v, b v$ and bu of sample $v b u$, at the Q-band. Data in the $a c^{*}$ and bu planes are drawn together. Red and blue colors distinguish sites $A$ and $B$. The solid lines are obtained with a fit of eqn (6) to the data, which is amplified in (f) for the case of the field along the $\boldsymbol{a}$ direction in the ba plane.
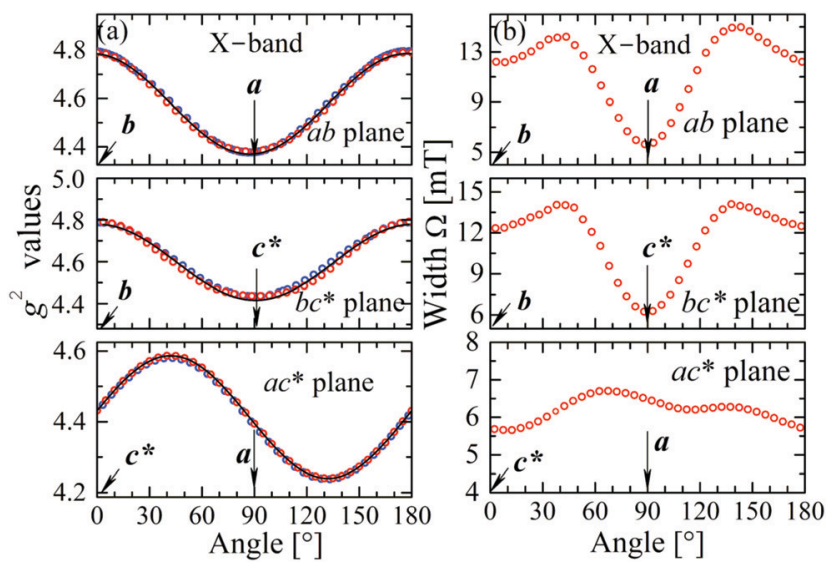

Fig. 7 Angular variation of (a) the $\boldsymbol{g}^{\mathbf{2}}$ factor and (b) the line width $\Gamma$ for $\boldsymbol{B}_{0}$ in the crystal planes $b a, b c^{*}$ and $a c^{*}$ of sample $a b c^{*}$, at the X-band. The solid lines are obtained from a fit of a $\boldsymbol{g}^{2}$-matrix (eqn (3)) to these data and the matrix elements of $\boldsymbol{g}^{2}$ are given in Table 3 .

around the crossing points (a factor of 8 between specific field orientations), respectively. According to our discussion in the experimental section, any effect of the temperature is negligible when compared with the effect of angular variation in the line width (Fig. 6). The spectra of a single crystal obtained at the $\mathrm{X}$-band from sample $\boldsymbol{a} \boldsymbol{a} \boldsymbol{c}^{*}$ show only one peak for all orientations of $\boldsymbol{B}_{0}$. The $g^{2}$-factor and width $\Omega$ obtained as for the Q-band spectra, but with one peak, are displayed in Fig. $7 \mathrm{a}$ and $\mathrm{b}$.

3.3.2 Powder samples. Fig. 8 displays the spectra observed from powder samples at X- (a), and Q-bands (c). Fig. 8d-f display simulations performed as described below.

\section{Exchange-coupled spins in a crystal lattice}

\subsection{Superexchange interactions}

Anderson ${ }^{68,69}$ explained formally the "superexchange" interaction between pairs of spins connected by a non-magnetic path introduced

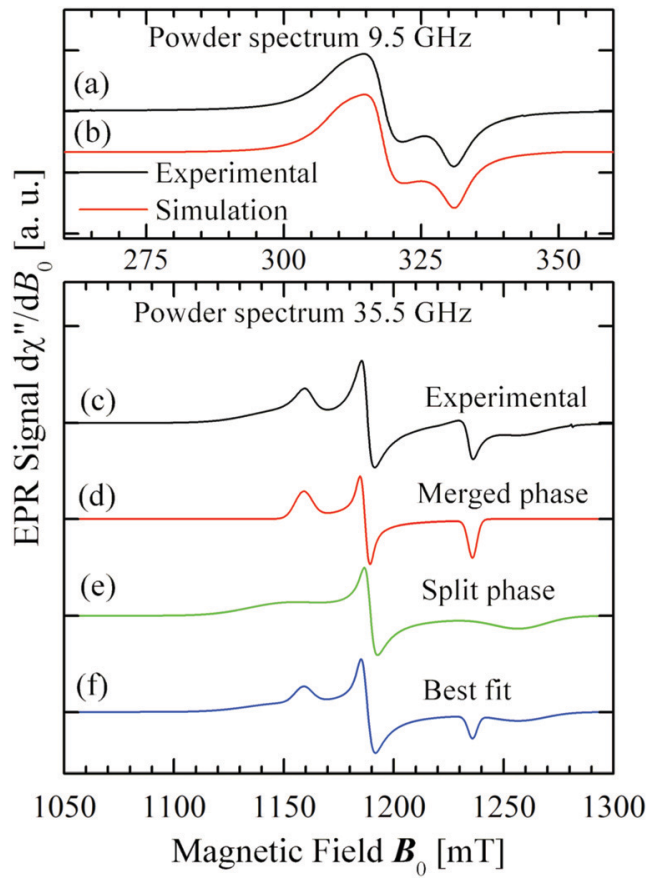

Fig. 8 (a) Observed spectrum of a powder sample at the X-band. (b) Best fit to the powder spectrum at the X-band (parameters in Table 3). (c) Observed spectrum of a powder sample at the Q-band. (d) and (e) Display simulations of the spectrum corresponding to each of the two phases. (f) Is the linear combination of the weighed components of (d) and (e) that best fit the experimental result.

by Kramers. ${ }^{70}$ His theory was enriched by Goodenough ${ }^{71}$ and Kanamori, ${ }^{72}$ who reported useful rules to calculate the magnitudes of these couplings. Years later, Hay et al. ${ }^{73}$ and $\mathrm{Kahn}^{74}$ added new theoretical concepts which are frequently used nowadays. Satisfactory agreement is obtained with these procedures between experimental and calculated exchange parameters in structures where the chemical paths supporting the superexchange are simple covalent bonds. ${ }^{74}$ Great difficulties appear, however, in cases where the paths are very long, non-covalent or a combination of various segments which may interfere or contribute differently to the actual magnitude of the coupling. These weak couplings connecting metal ions or strongly coupled polymetallic molecules introduce spin dynamics and important quantum effects in molecular magnets ${ }^{6,75}$ and for quantum information processing ${ }^{76,77}$ that can be studied empirically in structurally characterized materials, and EPR is very appropriate for this purpose.

\subsection{Chemical paths for superexchange in CuBMB}

The space group $P 2_{1} / c$ of CuBMB contains four symmetry related molecules $\mathrm{M}_{1}$ to $\mathrm{M}_{4} \equiv \mathrm{C}_{20} \mathrm{H}_{19} \mathrm{ClCuN}_{4} \mathrm{OS}$ (shown in Fig. 2) per unit cell, with atoms at $(x, y, z),(1 / 2-x, 1 / 2+y, 1 / 2-z),(-x,-y,-z)$ and $(1 / 2-x, 1 / 2+y, 1 / 2-z) . \mathrm{M}_{2}$ is obtained from $\mathrm{M}_{1}$ by a $180^{\circ}$ rotation around $b ; \mathrm{M}_{3}$ from $\mathrm{M}_{1}$ by an inversion, and $\mathrm{M}_{4}$ from $\mathrm{M}_{1}$ by a $180^{\circ}$ rotation plus an inversion. There are many long paths for superexchange interactions between copper ions but, considering the short range of the coupling, it is appropriate to consider only the shortest ones connecting nearest neighbours. Fig. 9 (central) displays a projection of the central molecular core $\mathrm{CuSON}_{3} \mathrm{~N}_{4} \mathrm{Cl}$ of $\mathrm{M}_{1}$ plus 


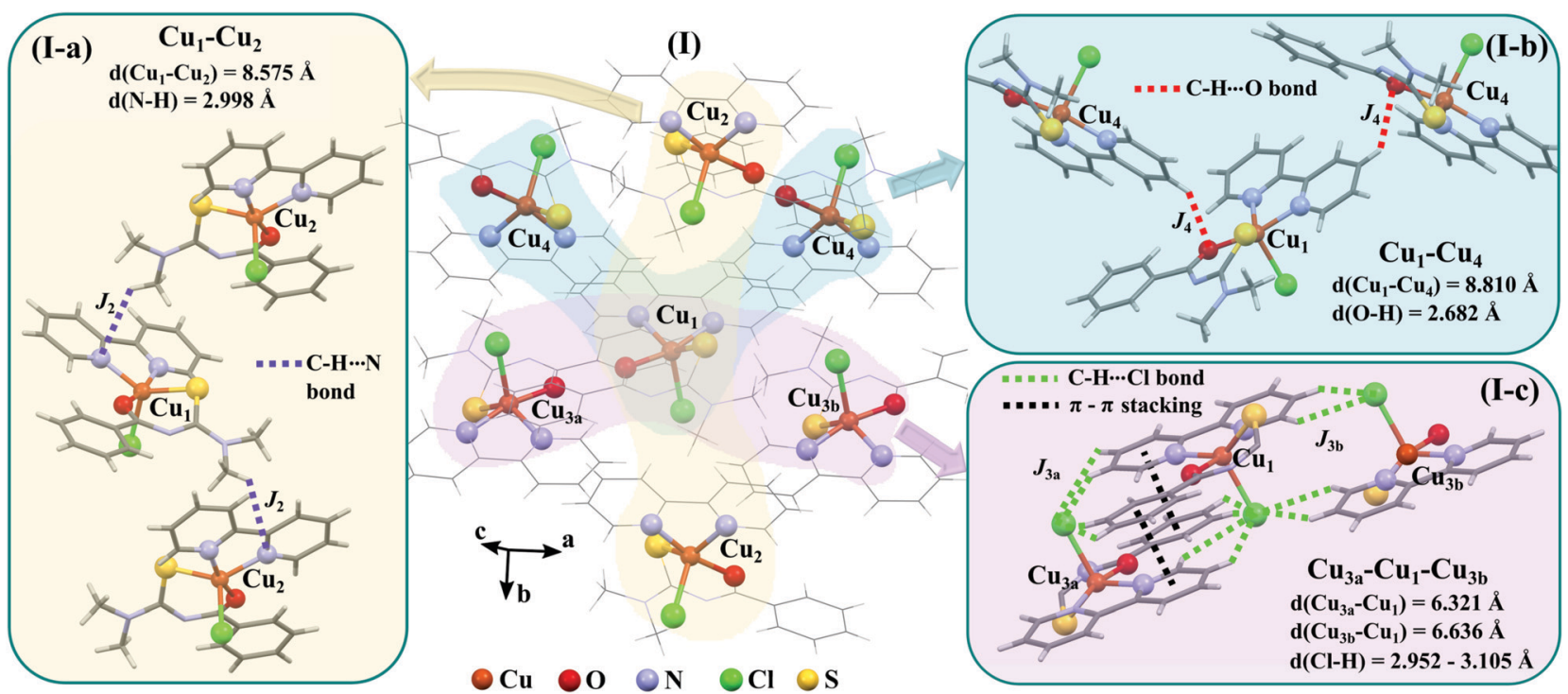

Fig. 9 (I) Three-dimensional array of a central molecule of CuBMB and the six neighbour molecules with $\mathrm{Cu}-\mathrm{Cu}$ distances shorter than $10 \AA$. . For clarity, the central molecular cores $\mathrm{CuOSN}{ }_{3} \mathrm{~N}_{4} \mathrm{Cl}$ are described with balls and sticks, and the remaining bonds as sticks. The subscripts of the Cu ions indicate the asymmetric units described in the text. (I-a) Chemical paths connecting $\mathrm{Cu}_{1}$ and $\mathrm{Cu}_{2}$, supporting exchange couplings $\mathrm{J}_{2}\left(z_{2}=2\right)$. (I-b) $\mathrm{Chemical}$ paths connecting $\mathrm{Cu}_{1}$ and $\mathrm{Cu}_{4}$ supporting $\mathrm{J}_{4}\left(z_{4}=2\right)$. (I-c) Chemical paths connecting $\mathrm{Cu}_{1}$ with $\mathrm{Cu}_{3 a}\left(z_{3 a}=1\right)$ and $\mathrm{Cu}_{3 b}\left(z_{3 b}=1\right)$, supporting $J_{3 a}$ and $J_{3 b}$, respectively.

those corresponding to the six nearest molecules with coppercopper distances shorter than $10 \AA$, which may be grouped by pairs of molecules $\mathrm{M}_{\mathrm{i}}$ containing coppers $\mathrm{Cu}_{\mathrm{i}}$, as the shortest paths between copper neighbours. Each $\mathrm{Cu}_{1}$ atom is equally connected to two $\mathrm{Cu}_{2}$ atoms at $8.575 \AA$ along equatorial-equatorial uniform zig-zag chains $-\mathrm{Cu}_{1}-\mathrm{Cu}_{2}-\mathrm{Cu}_{1}-\mathrm{Cu}_{2}-$ running in the $b$-direction with uniform coupling $\boldsymbol{J}_{2}$, connected by chemical paths having six diamagnetic atoms $\mathrm{P}_{1-2}=\mathrm{Cu}_{1}-\mathrm{S}-\mathrm{C} 8-\mathrm{N} 2-\mathrm{C} 9-\mathrm{H} \cdots \mathrm{N} 3-\mathrm{Cu}_{2}$ (Fig. 9(I-a)) with total length $12.97 \AA$ along paths including $\mathrm{H}$-bonds $\mathrm{C}-\mathrm{H} \cdots \mathrm{N}$ (underlined) between a methyl carbon of the $\mathrm{L}$ ligand to one copper, and a $\mathrm{N}$ of the bipy ligand of the following copper. There are two closest $\mathrm{Cu}_{3}$ neighbours to a $\mathrm{Cu}_{1}$ related by inversion centres at $6.321 \AA\left(\mathrm{Cu}_{3 \mathrm{a}}\right)$ and $6.636 \AA$ $\left(\mathrm{Cu}_{3 \mathrm{~b}}\right)$ in Fig. 9(I-c). They give rise to chains $-\mathrm{Cu}_{1}-\mathrm{Cu}_{3}-\mathrm{Cu}_{1}-$ $\mathrm{Cu}_{3}$ - along the $a$-direction, connected with alternate paths $\mathrm{P}_{1-3, a}=\mathrm{Cu}_{1}-\mathrm{C} 1-\mathrm{H} 31-\mathrm{C} 31-\mathrm{N} 3-\mathrm{Cu}_{3}$, with total length $9.88 \AA$, and $\mathrm{P}_{1-3, b}=\mathrm{Cu}_{1}-\mathrm{Cl}-\mathrm{H} 41-\mathrm{C} 41-\mathrm{N} 4-\mathrm{Cu}_{3}$, with total length $9.67 \AA$, both containing four similar diamagnetic atoms and including hydrogen bonds $\mathrm{C}-\mathrm{H} \cdots \mathrm{Cl}$ connecting apical $\mathrm{Cl}$ atoms of one $\mathrm{Cu}$ with bipy carbons of the other. Alternate couplings $\boldsymbol{J}_{\mathbf{3} a}$ and $\boldsymbol{J}_{\boldsymbol{3} \boldsymbol{b}}$ are different because $\mathrm{P}_{1-3, a}$ includes an important stacking of bipy rings of $\mathrm{Cu}_{1}$ and $\mathrm{Cu}_{3}$ at $\sim 3.5 \AA$, and none appears in $\mathrm{P}_{1-3, b}$. A third pair of connections of $\mathrm{Cu}_{1}$ (see Fig. 9(I-b)) is with two $\mathrm{Cu}_{4}$ at $8.810 \AA\left(\mathrm{Cu}_{1}\right.$ and $\mathrm{Cu}_{4}$ are related by a $180^{\circ}$ rotation plus an inversion), giving rise to zig-zag chains. These equatorial-equatorial paths $\mathrm{Cu}_{1}-\mathrm{N} 4-\mathrm{C}-\mathrm{C}-\mathrm{C} 43-\mathrm{H} 43-\mathrm{O}-\mathrm{Cu}_{4}$ involve 6 diamagnetic atoms including a bipy ring and a $\mathrm{C}-\mathrm{H} \cdots \mathrm{O}$ bond, with total length $11.68 \AA$ along the bond (Fig. 9(I-b)). Copper neighbours of the same type (as $\left.\mathrm{Cu}_{1}-\mathrm{Cu}_{1}\right)$ at distances equal to or longer than $a=11.979 \AA$ are ignored, as are also ignored the interactions with second $\mathrm{Cu}$ neighbours of all types that generate distributions of the couplings around the central values. The couplings $J_{2}$ and $J_{4}$ occur with two symmetrical neighbours $\left(z_{2}=z_{4}=2\right)$. Obviously, each of the four symmetry related coppers in the lattice has the same neighbourhood described for $\mathrm{Cu}_{1}$. In summary, the paths connecting copper ions in CuBMB are long and complex including both H-bonds and $\pi$-stacking.

\subsection{Lattice symmetry and spin Hamiltonian description}

Each $\mathrm{Cu}$ type in CuBMB gives rise to a spin-sublattice and to an EPR spectrum which depends on the properties of the site. Since coppers differing by an inversion operation are magnetically equal, we consider only two different sublattices, $\mathrm{A}$, containing $\mathrm{Cu}_{1}$ and $\mathrm{Cu}_{3}$, and $\mathrm{B}$, containing $\mathrm{Cu}_{2}$ and $\mathrm{Cu}_{4}$, whose spectra differ by a $\mathrm{C}_{2}$ rotation around $\boldsymbol{b}$. The main contributions to the spin Hamiltonian $\mathcal{H}_{0}$ are the Zeeman interaction with the field $\boldsymbol{B}_{0}$, and the isotropic intrasublattice and intersublattice exchange interactions $J_{i j}^{\alpha \beta}\left(\left|J_{i}\right| \ll h \nu\right)$ between $\mathrm{Cu}$ neighbours in the same or in different sublattices, written as:

$$
\begin{aligned}
\mathcal{H}_{0}= & \mu_{\mathrm{B}} \sum_{\alpha=A, B} \boldsymbol{B}_{0} \cdot \boldsymbol{g}_{\alpha} \cdot \sum_{i} \boldsymbol{S}_{i \alpha}-\sum_{\alpha=A, B} \sum_{i \neq j} J_{i j}^{\alpha \alpha} \boldsymbol{S}_{i \alpha} \cdot \mathbf{S}_{j \alpha} \\
& -\sum_{\alpha \neq \beta} \sum_{i, j} J_{i j}^{\alpha \beta} \boldsymbol{S}_{i \alpha} \cdot \boldsymbol{S}_{j \beta}+\mathcal{H}_{\mathrm{ss}}+\mathcal{H}_{\mathrm{hyp}}
\end{aligned}
$$

where $\boldsymbol{g}_{\alpha}$ is the $\boldsymbol{g}$-matrix of $\mathrm{Cu}$ ions in the sublattice $\alpha$ and $\boldsymbol{S}_{i \alpha}$ is the spin of the $\mathrm{Cu}_{\alpha}$ in the unit cell $i$. The sums of exchange terms are over the six closest neighbours. $\mathcal{H}_{0}$ also includes anisotropic spin-spin terms $\mathcal{H}_{\text {ss }}$ (mainly dipole-dipole interactions which are not detailed in eqn (1)) and hyperfine couplings $\mathcal{H}_{\text {hyp }}$ with the copper nuclei (not observed). At each field orientation $\boldsymbol{\eta}=\boldsymbol{B}_{0} /\left|\boldsymbol{B}_{0}\right|=$ $(\sin \theta \cos \phi, \sin \theta \sin \phi, \cos \theta$ ), where $\theta$ and $\phi$ are the equatorial and azimuthal angles in the laboratory coordinate system $x y z$ of Fig. 1 , 
the sublattices A and B give rise to EPR peaks at $g_{\alpha}=\left(\boldsymbol{\eta} \cdot \boldsymbol{g}_{\alpha} \cdot \boldsymbol{g}_{\alpha} \cdot \boldsymbol{\eta}\right)^{1 / 2},{ }^{30}$ and the spectrum of a single crystal is the sum of the spectra of the sublattices modified by the exchange coupling. To analyse these modifications, we write the Zeeman contributions to eqn (1) for $\alpha=\mathrm{A}$ and $\mathrm{B}$ as:

$$
\begin{aligned}
& \sum_{i} \mu_{\mathrm{B}} \boldsymbol{B}_{0} \cdot\left(\boldsymbol{g}_{\mathrm{A}} \cdot \boldsymbol{S}_{i \mathrm{~A}}+\boldsymbol{g}_{\mathrm{B}} \cdot \boldsymbol{S}_{i \mathrm{~B}}\right)= \\
& \sum_{i}\left[\mu_{\mathrm{B}} \boldsymbol{B}_{0} \cdot \boldsymbol{g} \cdot\left(\boldsymbol{S}_{i \mathrm{~A}}+\boldsymbol{S}_{i \mathrm{~B}}\right)+\mu_{\mathrm{B}} \boldsymbol{B}_{0} \cdot \boldsymbol{G} \cdot\left(\boldsymbol{S}_{i \mathrm{~A}}-\boldsymbol{S}_{i \mathrm{~B}}\right)\right]
\end{aligned}
$$

where $g=1 / 2\left(g_{\mathrm{A}}+g_{\mathrm{B}}\right)$ and $\boldsymbol{G}=1 / 2\left(g_{\mathrm{A}}-g_{\mathrm{B}}\right)$. For magnetically equal neighbour pairs as occurs for $\mathrm{Cu}_{1}-\mathrm{Cu}_{3}$ (see Fig. 9c), $\boldsymbol{G}=0$ and the second Zeeman contribution to eqn (2) cancels. Since intrasublattice exchange interactions with magnitudes $J_{3 a}$ and $J_{3 b}$ commute with the total spin of the sublattice, they do with the Zeeman interaction, and do not change the positions of the resonances. However, these terms do not commute with the anisotropic spin-spin interaction $\mathcal{H}_{\text {ss }}$, and modify the widths of the peaks. Intersublattice exchange interactions such as $J_{2}$ and $J_{4}$ arising from the contacts shown in Fig. 9(I-a) and (I-b) modulate and average out terms proportional to $\boldsymbol{G} \neq 0$, producing additional changes to the widths and modifying the positions of the peaks. ${ }^{17}$ So, different sources of narrowing and shifting the resonances provide complementary information. Both coupling pairs $J_{3 a}$ and $J_{3 b}$, and $J_{2}$ and $J_{4}$ produce equal effects and cannot be distinguished experimentally. The two EPR peaks merge to one peak when the intersublattice exchange coupling becomes equal to the anisotropic Zeeman contribution to eqn (2) proportional to $G=1 / 2\left(g_{\mathrm{A}}-g_{\mathrm{B}}\right)$. So, when the intersublattice exchange coupling is negligible compared with the anisotropic Zeeman contribution proportional to $\boldsymbol{G}$ for a particular orientation of $\boldsymbol{B}_{0}$, the full Zeeman interaction of eqn (1) applies, and we observe as a function of angle two resonances with $g$-factors $g_{\mathrm{A}}$ and $g_{\mathrm{B}}$, allowing us to calculate from these data the matrices $\boldsymbol{g}_{\mathrm{A}}{ }^{2}$ and $\boldsymbol{g}_{\mathrm{B}}{ }^{2}$. Instead, if the exchange coupling between sublattices A and B is larger than the Zeeman splitting this is averaged out and we observe a single resonance with an angle dependent $g$-factor $g=1 / 2\left(g_{\mathrm{A}}+g_{\mathrm{B}}\right)$ that allows us to evaluate the components of the matrix $g^{2}=1 / 2\left(g_{\mathrm{A}}{ }^{2}+g_{\mathrm{B}}{ }^{2}\right)$. There are field orientations where the resonances are merged, and others where they are split, and a transition between these situations is produced by varying the orientation of $\boldsymbol{B}_{0}$, as shown in Fig. 5. In addition, strong changes of the line width $\Omega$ are observed in the merged ranges (Fig. 6).

\section{Calculated results}

\subsection{Evaluation of the $g^{2}$-matrices of the $\mathrm{Cu}^{\mathrm{II}}$ ions}

From the full set of data in each of the two regimes we calculate the components of the matrices $g^{2}$, or $g_{\mathrm{A}}{ }^{2}$ and $\boldsymbol{g}_{\mathrm{B}}{ }^{2}$ by global least-squares fits of the function: ${ }^{78}$

$$
\begin{aligned}
g^{2}(\theta, \phi)= & \boldsymbol{\eta} \cdot \boldsymbol{g} \cdot \boldsymbol{g} \cdot \boldsymbol{\eta}=\left(g^{2}\right)_{x x} \sin ^{2} \theta \cos ^{2} \phi+\left(g^{2}\right)_{y y} \sin ^{2} \theta \sin ^{2} \phi \\
& +\left(g^{2}\right)_{z z} \cos ^{2} \theta+2\left(g^{2}\right)_{x y} \sin ^{2} \theta \sin \phi \cos \phi \\
& +2\left(g^{2}\right)_{x z} \sin \theta \cos \theta \cos \phi+2\left(g^{2}\right)_{y z} \sin \theta \cos \theta \sin \phi
\end{aligned}
$$

to data in each regime. It has to be noted that the exchange couplings average out the $g$-values of the two peaks but the experimental results are the values of $g^{2}$, a condition that has to be considered in the calculations. ${ }^{79,80}$ The matrix elements of $\boldsymbol{g}_{\mathrm{A}}{ }^{2}$ and $\boldsymbol{g}_{\mathrm{B}}{ }^{2}$ were obtained by fitting data at the Q-band in angular regions of Fig. 5 where the peaks are well split or the collapse is due to symmetry conditions. ${ }^{26}$ As the spectra collected in fully-split angular ranges are few, we obtained the components of $\boldsymbol{g}_{\mathrm{A}}{ }^{2}$ and $\boldsymbol{g}_{\mathrm{B}}{ }^{2}$ as those producing lines tangent to the experimental results in Fig. 5. Besides, the data in the $b v$ plane (see Fig. 5), where the peaks A and B are fully collapsed, not as a consequence of a symmetry condition as in the ac plane, but because two contributions cancel out, were particularly useful to gain accuracy in these fits, and provided an important contribution of the data for sample $\boldsymbol{u} \boldsymbol{b v}$ of Fig. 1. The fit of the data in Fig. 5 in angular regions where the peaks are fully collapsed allows obtaining the matrix elements of $g^{2}=1 / 2\left(g_{\mathrm{A}}^{2}+g_{\mathrm{B}}{ }^{2}\right)$, according to the justification given before. Equal results for the matrix elements of $\boldsymbol{g}^{2}$ were obtained by averaging the experimental values of $\boldsymbol{g}_{\mathrm{A}}{ }^{2}$ and $\boldsymbol{g}_{\mathrm{B}}{ }^{2}$ at the Q-band and fitting them with eqn (3). Another set of components of the $\boldsymbol{g}^{2}$-matrix was obtained by fitting eqn (3) to the results in Fig. 7 at the X-band, where the resonances are merged for all field orientations. The components of $\boldsymbol{g}^{2}, \boldsymbol{g}_{\mathrm{A}}{ }^{2}$ and $\boldsymbol{g}_{\mathrm{B}}{ }^{2}$ are given in Table 3 and the calculated values are shown with solid lines

\begin{tabular}{|c|c|c|c|c|c|c|c|}
\hline Matrix elements & & $\left(g^{2}\right)_{x x}$ & $\left(g^{2}\right)_{y y}$ & $\left(g^{2}\right)_{z z}$ & $\left(g^{2}\right)_{x y}$ & $\left(g^{2}\right)_{x z}$ & $\left(g^{2}\right)_{y z}$ \\
\hline Single crystal Q-band (global fit of split-phase) & $\begin{array}{l}g_{\mathrm{A}}^{2} \\
g_{\mathrm{B}}^{2}\end{array}$ & $\begin{array}{l}4.357(1) \\
4.357(1)\end{array}$ & $\begin{array}{l}4.421(1) \\
4.421(1)\end{array}$ & $\begin{array}{l}4.774(1) \\
4.774(1)\end{array}$ & $\begin{array}{l}0.175(1) \\
0.175(1)\end{array}$ & $\begin{array}{r}0.210(1) \\
-0.210(1)\end{array}$ & $\begin{array}{r}-0.240(1) \\
0.240(1)\end{array}$ \\
\hline Single crystal Q-band (global fit of merged-phase) & $\begin{array}{l}\boldsymbol{g}_{\mathrm{A}}, \boldsymbol{g}_{\mathrm{B}} \text { eigenvalues } \\
\boldsymbol{g}^{2} \\
\boldsymbol{g} \text { eigenvalues }\end{array}$ & $\begin{array}{l}2.017 \\
4.357(1) \\
2.052\end{array}$ & $\begin{array}{l}2.103 \\
4.421(1) \\
2.137\end{array}$ & $\begin{array}{l}2.218 \\
4.774(1) \\
2.185\end{array}$ & $0.175(1)$ & 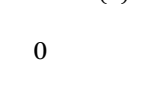 & 0 \\
\hline Single crystal X-band & 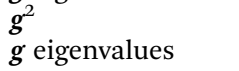 & $\begin{array}{l}4.358(1) \\
2.052\end{array}$ & $\begin{array}{l}4.424(1) \\
2.137\end{array}$ & $\begin{array}{l}4.775(1) \\
2.185\end{array}$ & $0.168(1)$ & 0 & 0 \\
\hline
\end{tabular}
in Fig. 5 and 7a. The good agreement between the values obtained from the different sources of data confirms the hypotheses made. The FWHM line widths $\Omega$ observed in the six studied crystal planes

Table 3 Components of the $\boldsymbol{g}^{2}$-matrices obtained from EPR data for single crystal and powder samples at Q and X-bands shown in Fig. 5-8

Matrix elements

Powder, X-band

Powder, Q-band ${ }^{\mathrm{i}}$

Powder, Q-band ${ }^{\text {ii }}$

DFT prediction

$\begin{array}{llll} & \boldsymbol{g}_{\mathbf{1}} & \boldsymbol{g}_{\mathbf{2}} & \boldsymbol{g}_{\mathbf{3}} \\ \text { Merged-phase } & 2.052 & 2.136 & 2.189 \\ \text { Merged-phase } & 2.052 & 2.136 & 2.189 \\ \text { Split-phase } & 2.020 & 2.135 & 2.218 \\ \text { Single molecule } & 2.022 & 2.152 & 2.194\end{array}$


at the Q-band and the three planes at the X-band (Fig. 6 and $7 \mathrm{~b}$ ) are the other piece of experimental information. There is a nearly isotropic residual width of $\sim 5.8 \mathrm{mT}$ (weakly dependent on temperature) similar at both bands, and strong angular variations in the $b a, b c^{*}$ and $b v$ planes, with widths that narrow by a factor of up to $\sim 8$ at the Q-band with minima (residual width) in the axes $\boldsymbol{a}$, $\boldsymbol{b}, \boldsymbol{c}^{*}$ and $\boldsymbol{u}$ in the planes $b a, b c^{*}$ and $b u$, analysed below. The relations of the eigenvalues and eigenvectors of $\boldsymbol{g}^{2}, \boldsymbol{g}_{\mathrm{A}}^{2}$ and $\boldsymbol{g}_{\mathrm{B}}{ }^{2}$ with the structure and the electronic wave functions of the copper ions in CuBMB are modelled below.

\subsection{Evaluation of the exchange parameters}

5.2.1 Single-crystal data. We evaluate here the exchange interactions $J_{i}$ from the data in Fig. 5 and 6 using basic results of Anderson's theory. ${ }^{12,13}$ We do that by plotting in Fig. 10a-f the ratio $R=\delta B /\left|\delta B_{0}\right|$ between the observed and calculated (from the values of $\boldsymbol{G}$ ) field distances $\delta B$ and $\delta B_{0}$ between the two split peaks, as a function of the modulus of the calculated distances for $\boldsymbol{B}_{0}$ in the neighbourhood of the $\boldsymbol{a}$ and $\boldsymbol{b}$ axes in the $b a$ plane, the $\boldsymbol{b}$ and $\boldsymbol{c}^{*}$ axes

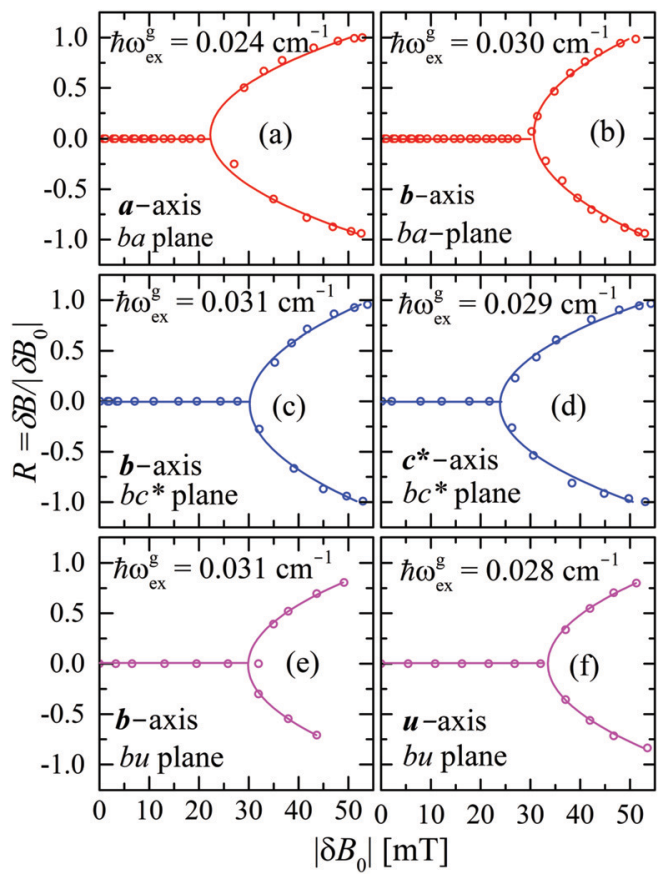

Fig. 10 Ratio $R=\delta B /\left|\delta B_{0}\right|$ in which $\delta B$ are the observed field distances and $\left|\delta B_{0}\right|$ are the field distances calculated without merging as a function of $\left|\delta B_{0}\right|$ measured with the field $\boldsymbol{B}_{0}$ around the axes in the $b a, b c$ and $b u$ planes. Solid lines are obtained from the fit of eqn (5) to the data. in the $b c^{*}$ plane and the $\boldsymbol{b}$ and $\boldsymbol{u}$ axis in the $b u$ plane. $R=0$ corresponds to fully merged peaks, and $R=1$ to fully split peaks. The observed curves display transition points between two and one peaks where the merging becomes complete. The results in Fig. 10 are explained ${ }^{21,26}$ in terms of the exchange frequency $\omega_{\mathrm{ex}}^{\mathrm{g}}$, associated with spin correlation functions related to the merging process, and thus with the exchange couplings $J_{i}$ connecting rotated spins as, ${ }^{12,26}$

$$
R=\frac{\delta B}{\left|\delta B_{0}\right|}=\frac{\Delta g_{\text {exp }}}{\Delta g_{\text {calc }}}= \pm \sqrt{1-\left(\frac{\hbar \omega_{\mathrm{ex}}^{\mathrm{g}}}{\mu_{\mathrm{B}} \Delta g_{\text {calc }} B_{0}}\right)^{2}}
$$

in agreement with the result in Fig. 10, and allowing us to obtain $\hbar \omega \omega_{\mathrm{ex}}^{\mathrm{g}}$ from the merging condition as:

$$
\hbar \omega_{\mathrm{ex}}^{\mathrm{g}}=\mu_{\mathrm{B}}\left|\Delta g_{\text {calc }}\right| B_{0}=\frac{\left|\Delta g_{\text {calc }}\right|}{g} h \nu
$$

where $\Delta g_{\text {calc }}(\theta, \phi)=g_{\mathrm{A}}(\theta, \phi)-g_{\mathrm{B}}(\theta, \phi)=\sqrt{\boldsymbol{\eta} \cdot \boldsymbol{g}_{\mathrm{A}}^{2} \cdot \boldsymbol{\eta}}-\sqrt{\boldsymbol{\eta} \cdot \boldsymbol{g}_{\mathrm{B}}^{2} \cdot \boldsymbol{\eta}}$, $g(\theta, \phi)=\sqrt{\boldsymbol{\eta} \cdot \boldsymbol{g}^{\mathbf{2}} \cdot \boldsymbol{\eta}}$ and the calculated values of $\hbar \omega_{\mathrm{ex}}^{\mathrm{g}}$ along the directions $\boldsymbol{\eta}$ where the full mergings are reached in Fig. 5 are given in Table 4.

In Fig. 11a-f we plot the FWHM width $\Omega$ as a function of the calculated distance $\left|\delta B_{0}\right|$ to the central position, for each central direction. Following Anderson, ${ }^{12,13}$ Passeggi and Calvo ${ }^{21}$ proposed that in the merged phase, the FWHM is given by the relation:

$$
\Omega=\frac{\sqrt{2 \pi}}{g \mu_{\mathrm{B}}} \frac{\left[\Delta g_{\text {calc }} \mu_{\mathrm{B}} B_{0}\right]^{2}}{\hbar \omega_{\mathrm{ex}}^{\Omega}}+\Omega_{0}=\frac{\sqrt{2 \pi}(h \nu)^{2}}{g \mu_{\mathrm{B}} \hbar \omega_{\mathrm{ex}}^{\Omega}} \frac{\left(\Delta g_{\text {calc }}\right)^{2}}{g^{2}}+\Omega_{0}
$$

where $\omega_{\mathrm{ex}}^{\Omega}$ is associated with spin correlation functions related to the narrowing process and $\Omega_{0}$ considers contributions to $\Omega$ arising from other mechanisms that are nearly isotropic, as shown in the $a c$ and $u v$ planes of Fig. $6 \mathrm{c}$ and d. Eqn (6) predicts a width proportional to the square of the microwave frequency $\nu$ and the factor $\sqrt{2 \pi}$ considers that we measure FWHM widths of Lorentzian derivative signals. ${ }^{21}$ The data were fitted to the quadratic dependence on $\Delta g_{\text {calc }}$ (solid lines in Fig. $6 \mathrm{a}$, b and e, enlarged in Fig. $6 \mathrm{f}$ and Fig. 11a-f), and the calculated values of $\omega_{\text {ex }}^{\Omega}$ are collected in Table 4 .

The relations between $\omega_{\mathrm{ex}}^{\mathrm{g}}$ and $\omega_{\mathrm{ex}}^{\Omega}$, and the intra- and intersublattice exchange parameters are:

$$
\begin{gathered}
\hbar \omega_{\mathrm{ex}}^{\mathrm{g}}=\sqrt{z_{2} J_{2}^{2}+z_{4} J_{4}^{2}} \\
\hbar \omega_{\mathrm{ex}}^{\Omega}=\sqrt{z_{2} J_{2}^{2}+J_{3 a}^{2}+J_{3 b^{2}+z_{4} J_{4}^{2}}}
\end{gathered}
$$

where $z_{2}=z_{4}=2$ are the numbers of $\mathrm{Cu}_{2}$ and $\mathrm{Cu}_{4}$ neighbours of a $\mathrm{Cu}_{1}$. Eqn (7) and (8) allow estimating the magnitudes of the

Table 4 Exchange frequencies $\omega_{\mathrm{ex}}$ obtained from EPR data at the Q-band considering the collapse observed in Fig. 10a-f ( $\left.\omega_{\mathrm{ex}}^{\mathrm{g}}\right)$, and widths of the resonances in Fig. 11a-f $\left(\omega_{\mathrm{ex}}^{\Omega}\right)$, using eqn (5) and (6). Combinations of exchange parameters $J_{i}$ calculated using eqn (9). The experimental uncertainties are $\sim 10 \%$

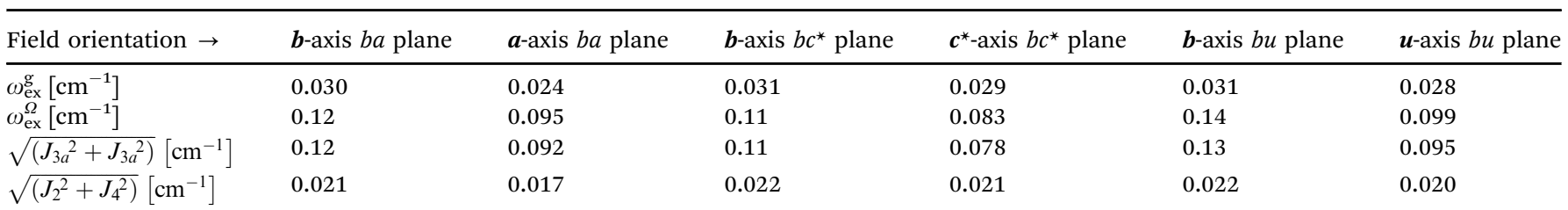




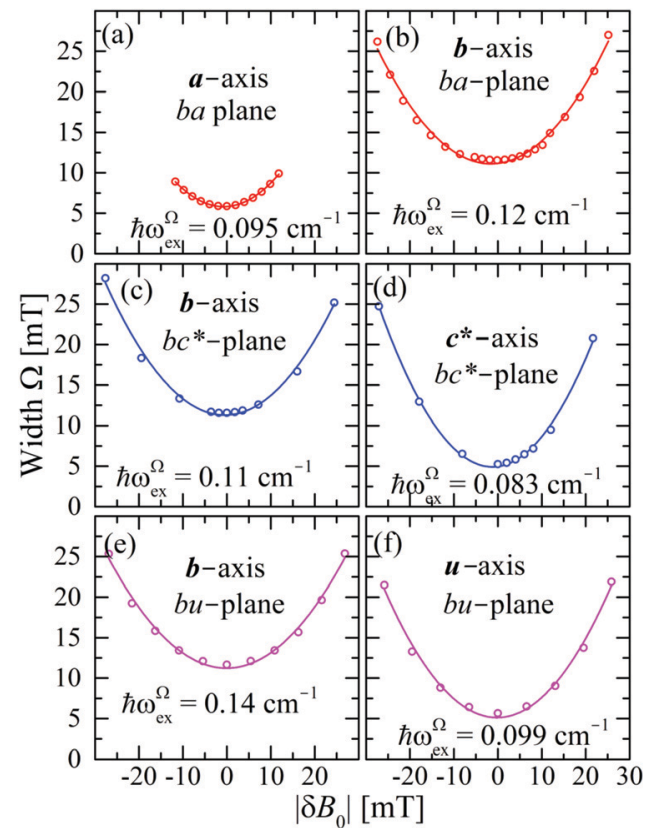

Fig. 11 FWHM width $\Omega$ as a function of $\left|\delta B_{0}\right|$, measured with the field $\boldsymbol{B}_{0}$ around the axes in the $b a, b c$ and bu planes. Lines are obtained from fits of eqn (8) to the data.

exchange couplings included in Table 4 from the measured exchange frequencies,

$$
J_{2}^{2}+J_{4}^{2}=1 / 2\left(\hbar \omega_{\mathrm{ex}}^{\mathrm{g}}\right)^{2} \text { and } J_{3 a}{ }^{2}+J_{3 b}{ }^{2}=\left(\hbar \omega_{\mathrm{ex}}^{\Omega}\right)^{2}-\left(\hbar \omega_{\mathrm{ex}}^{\mathrm{g}}\right)^{2}
$$

where no information about the signs of the exchange couplings $J_{i}$ is obtained.

5.2.2 Powder data. The spectrum $\mathrm{d} \chi^{\prime \prime} / \mathrm{d} B_{0}$ of a powder sample at the X-band (Fig. 8a) was simulated using EasySpin ${ }^{53}$ with the $g$-factors calculated from the single crystal study given in Fig. 8a and Table 3. We assumed that the widths of the peaks vary with angle as an ellipsoid with the same principal axes as the $g$-matrix, and optimised their principal values with a fit to the powder spectrum keeping fixed the $g$-factors obtained in the single crystal measurements. Clearly, the small differences between experimental (Fig. 7) and simulated X-band powder spectra (Fig. 8b) arise from the assumption about the angular dependence of the widths of the peaks. The spectrum at the Q-band (Fig. 8c) displays a novel result which to our knowledge has not been reported before. At this microwave frequency, the spectrum of a powder sample of spins 12 with a non-axially symmetric $\boldsymbol{g}$-matrix is expected to display three resolved peaks at the three eigenvalues of the $g$-matrix, $g_{1}<g_{2}<g_{3}{ }^{30}$ Instead, we observe (Fig. 8c) the sum of two spectra like this, with different intensities, values of $g_{1}$ and $g_{3}$ and widths. This is equivalent to having a sample containing two different spin species, as represented with the cartoon in Fig. 12, where green microcrystals and spectra are in the split-peak phase (having a larger field span and widths) and blue crystals and spectra are in the merged-peak phase (entangled-spins with smaller line widths, see inset (a) of Fig. 12). We proved that hypothesis by

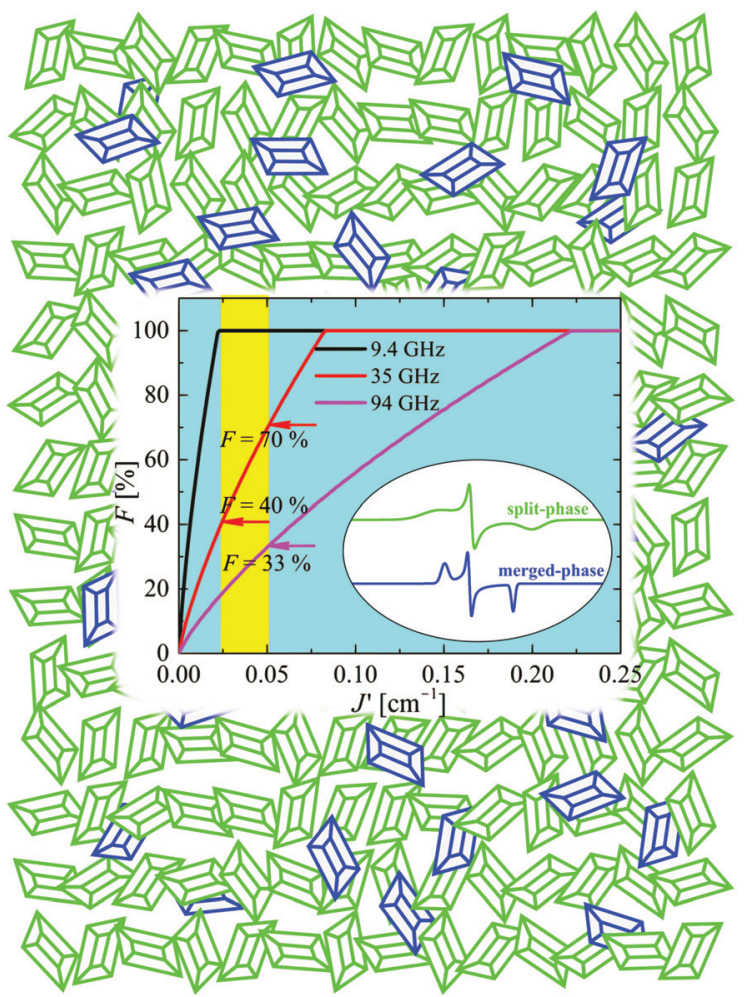

Fig. 12 Cartoon explaining the EPR spectrum of the powder (polycrystalline) sample of CuBMB at the Q-band with contributions arising from crystallites in two phases. Green microcrystals and spectra are in the split-signal phase; blue microcrystals and spectra are in the collapsed-signal phase. The sum of the integrated spectra (eqn (10)) fits the observed powder spectrum with $F \approx 0.28$ with a relatively large uncertainty due to background correction issues.

integrating the experimental result $\mathrm{d} \chi^{\prime \prime} / \mathrm{d} B_{0}$ to calculate the absorption powder spectrum $\chi^{\prime \prime}\left(B_{0}\right)$, and simulating it as the sum of absorption spectra of the merged and split phases with coefficients $F$ and $(1-F)$,

$$
\chi^{\prime \prime}(\operatorname{sim} .)=C\left[F \chi^{\prime \prime}(\text { merged-phase })+(1-F) \chi^{\prime \prime}(\text { split-phase })\right]
$$

We used the spin Hamiltonian parameters obtained from the powder spectrum at the X-band to simulate the merged phase spectrum, and parameters of the single crystal spectrum at the Q-band for the split phase (in this case the widths were obtained by optimizing the simulation of the spectrum in Fig. 8c). The factor $C$ in eqn (10) depends on the total mass of the powder sample, but the factor $F$ is the portion of the sample in the entangled phase, which depends on the couplings $\left|J_{i}\right|$ and on the microwave frequency, and is a consequence of the transition displayed in Fig. 10 and 11. The calculated spectra in the two phases are displayed in Fig. 8d and e and $F$ was obtained from a least squares fit of eqn (10) to the data. This fit indicates that at the Q-band $F=28 \%$ of the micro crystals are in the "merged-signal" phase, and $72 \%$ in the "split-signal" phase, and the spectrum simulated is shown in Fig. 8f. Instead, at the X-band, $\sim 100 \%$ of the micro crystals are in the merged-signal phase $(F \approx$ $100 \%$ ), and at higher microwave frequencies (e.g., $\nu \sim 94 \mathrm{GHz})$, 
the powder sample is expected to contain most micro crystals in the split-signal phase. This behaviour is displayed in the inset of Fig. 12, where we plot $F$ as a function of the average intersublattice exchange interactions at three microwave frequencies. We obtained these curves with a geometric model assuming that the microcrystals in the powder in which the distance between the resonance peaks is smaller than the average exchange coupling, $\left|g_{\mathrm{A}}(\theta, \phi)-g_{\mathrm{B}}(\theta, \phi)\right| \mu_{\mathrm{B}} B_{0} \leq\left|J_{i}\right|$, are in the merged ("blue") phase. We summed over a sphere the number of microcrystals in the merged phase for the case of CuBMB using the values of $g_{\mathrm{A}}(\theta, \phi)$ and $g_{\mathrm{B}}(\theta, \phi)$ calculated from the matrices in Table 3 over a large set (10 000) of equally spaced orientations $(\theta, \phi)$ obtained with the program Repulsion. ${ }^{81}$ Our result supports the added convenience of powder spectra to estimate exchange couplings in weakly coupled compounds. One should note that the experimental uncertainty is large because of baseline problems.

\subsection{DFT calculations}

The molecular reference system based on the electronic configuration is given by the axes $\left(X_{g}, Y_{g}, Z_{g}\right)$ corresponding to the principal directions of the $g$-matrix. The angles between the principal directions of the $\boldsymbol{g}$-matrix and the $\mathrm{Cu}$-ligand directions calculated both from EPR and DFT are in reasonable agreement and shown in Table 6. The directions $\left(X_{g}, Y_{g}, Z_{g}\right.$ ) obtained experimentally (black) and $\left(\left[X_{g}\right],\left[Y_{g}\right],\left[Z_{g}\right]\right)$ (gray) obtained numerically are depicted in Fig. 5f, where $X_{g}\left(\left[X_{g}\right]\right)$ is pointing close to the direction of the $\mathrm{Cu}-\mathrm{O}$ bond, $Y_{g}\left(\left[Y_{g]}\right]\right)$ is close to the $\mathrm{Cu}-\mathrm{S}$ bond, and $Z_{g}\left(\left[Z_{g}\right]\right)$ lies between the directions of the $\mathrm{Cu}-\mathrm{Cl}$ and $\mathrm{Cu}-\mathrm{S}$ bonds. The experimental and calculated principal values of the $\boldsymbol{g}$-matrix are in good agreement as shown in Table 4 . The $Z_{g}$ direction is canted from the $\mathrm{Cu}-\mathrm{Cl}$ bond direction and limits the description of the electronic structure based on square planar symmetry for the so called equatorial direction of the crystal structure. The calculated exchange parameters are shown in Table 5 and are in good agreement with the experimental values. The spin density plot is shown in Fig. 13 and supports the depicted exchange pathways with non-zero spindensities on the ligands. The output file is included with the ESI. $\dagger$

\section{Discussion}

\subsection{Electronic structure of isolated molecules}

Detailed EPR experiments in six crystal planes allowed us to evaluate the $\boldsymbol{g}^{2}$-matrix for $\mathrm{Cu}^{\mathrm{II}}$ sites in $\mathrm{CuBMB}$ which is related

Table 5 Exchange frequencies obtained via DFT-BS modeling for individual pairs of molecules according to the exchange pathways depicted in Fig. 9. Combinations of exchange parameters for comparison with the experimental values in Table 4

\begin{tabular}{lr}
\hline$J_{3 a}^{\mathrm{c}}\left[\mathrm{cm}^{-1}\right]$ & -0.06 \\
$J_{3 b}^{\mathrm{c}}\left[\mathrm{cm}^{-1}\right]$ & 0.10 \\
$J_{2}^{\mathrm{c}}\left[\mathrm{cm}^{-1}\right]$ & -0.02 \\
$J_{4}^{\mathrm{c}}\left[\mathrm{cm}^{-1}\right]$ & -0.03 \\
$\sqrt{\left[\left(J_{3 a}^{\mathrm{c}}\right)^{2}+\left(J_{3 b}^{\mathrm{c}}\right)^{2}\right]}\left[\mathrm{cm}^{-1}\right]$ & 0.12 \\
$\sqrt{\left[\left(J_{2}^{\mathrm{c}}\right)^{2}+\left(J_{4}^{\mathrm{c}}\right)^{2}\right]}\left[\mathrm{cm}^{-1}\right]$ & 0.04
\end{tabular}
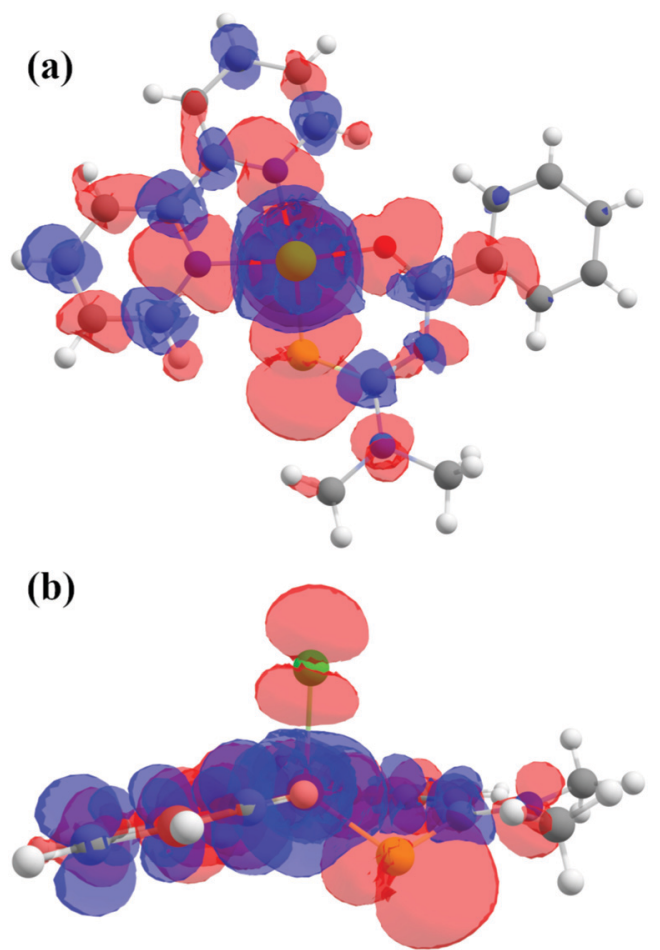

Fig. 13 (a) Spin density plot as calculated by DFT, viewed (a) perpendicular and (b) parallel to the plane of the rings. The electronic distribution is concentrated around the $\mathrm{Cu}$ ion, the bipy and the $\mathrm{Cl}$ ligands.

Table 6 Calculated angles $\left(^{\circ}\right)$ between the copper-ligand directions and the molecular orientations $\left(X_{g}, Y_{g}, Z_{g}\right)$ as determined by the direction of the $\boldsymbol{g}$-matrix from experiments and from numerical DFT calculations (indicated in square brackets). The directions are depicted in Fig. $5 f$

\begin{tabular}{llll}
\hline & $X_{g}$ & $Y_{g}$ & $Z_{g}$ \\
\hline $\mathrm{Cu}-\mathrm{Cl}$ & $76[87]$ & $55[60]$ & $39[30]$ \\
$\mathrm{Cu}-\mathrm{S}$ & $85[91]$ & $166[174]$ & $77[84]$ \\
$\mathrm{Cu}-\mathrm{O}$ & $8[6]$ & $83[89]$ & $86[84]$ \\
$\mathrm{Cu}-\mathrm{N} 1$ & $88[84]$ & $55[48]$ & $145[137]$ \\
$\mathrm{Cu}-\mathrm{N} 2$ & $162[173]$ & $98[91]$ & $106[97]$
\end{tabular}

to the electronic wavefunctions. The calculated eigenvectors $\left(X_{g}, Y_{g}, Z_{g}\right)$ show a molecular frame oriented in an uncommon orientation for a square pyramidal structure, indicating a highly distorted electronic structure which recalls the situation of the so-called blue proteins, in which a back donation effect is caused by the sulfur ligand, ${ }^{82,83}$ a situation rarely reported for small copper organic complexes like CuBMB. Further evidence of this uncommon result is offered by our DFT calculations that show relevant electron density around the Cl ligand. This result contrasts with that expected for an apical ligand in a more standard $\mathrm{Cu}^{\mathrm{II}}$ ion with a $\mathrm{d}_{x^{2}-y^{2}}$ ground orbital state and the $\mathrm{Cl}$ atom in an apical position.

\subsection{Intermolecular superexchange couplings}

We observe a dispersion of $\sim 20 \%$ for $\omega_{\mathrm{ex}}^{\mathrm{g}, \Omega}$ along rows of Table 4. This comes from simplifications of our approximate model for the $\left|J_{i}\right|$, that do not take into account the distribution 
of spin-spin interactions of each individual superexchange path (Fig. 9) but their averaged effect in the EPR spectra. Despite the dispersion, our proposed model for calculating $\left|J_{i}\right|$ is corroborated by the DFT results in Table 5 that were obtained considering the individual superexchange paths.

The values of the averages $\sqrt{J_{2}^{2}+J_{4}^{2}}$ given in Table 4 are the outcomes of the paths $\mathrm{P}_{1-2}$ and $\mathrm{P}_{1-4}$ described in Section 4.2. They contain 6 diamagnetic atoms and include H-bonds $\mathrm{C}-\mathrm{H} \cdots \mathrm{N}$ and $\mathrm{C}-\mathrm{H} \cdots \mathrm{O},{ }^{84,85}$ respectively. The other important difference is the replacement of a sulfur in the path $\mathrm{P}_{1-2}$ by a nitrogen in $\mathrm{P}_{1-4}$. Since the $\mathrm{C}-\mathrm{H} \cdots \mathrm{O}$ bond is shorter than the $\mathrm{C}-\mathrm{H} \cdots \mathrm{N}$ bond, $J_{4}$ may have a more significant contribution than $J_{2}$. We do not have experimental evidence to assess the specific roles of these differences in the supported exchange couplings $J_{2}$ and $J_{4}$, but the DFT results from Table 5 confirm that they are similar with $J_{4}^{\mathrm{c}}$ slightly greater than $J_{2}^{\mathrm{c}}$.

The larger values of the averages $\sqrt{J_{3 a^{2}}+J_{3 a^{2}}}$ compared to $\sqrt{J_{2}^{2}+J_{4}^{2}}$ are supported by the chemical paths $\mathrm{P}_{1-3_{a}}$ and $\mathrm{P}_{1-3_{b}}$ that contain only 4 diamagnetic atoms with a contribution of eight (for $J_{3 a}$ ) and four (for $J_{3 b}$ ) similar hydrogen bonds $\mathrm{C}-\mathrm{H} \cdots \mathrm{Cl}$ as observed in Fig. 9(I-c). We noted before that an important difference between these paths is the contribution of $\pi-\pi$-stacking of bipyridine-benzoyl rings to $J_{3 a}{ }^{86}$ In fact, from the exchange frequencies modeled by DFT-BS, the $\mathrm{P}_{1-3_{a}}$ path has an overall antiferromagnetic behaviour while $\mathrm{P}_{1-3_{b}}$ is ferromagnetic with $\left|J_{3 b}^{\mathrm{c}}\right|>\left|J_{3 a}^{\mathrm{c}}\right|$. It suggests that the stacking contribution is antiferromagnetic, since the $\mathrm{C}-\mathrm{H}$. . Cl bridges are similar in both paths. The work of Venegas et al. assigned to a similar coupling of bipyridine molecules in lamellar $\left[\mathrm{Cu} \text { (bipy)(en) } \mathrm{Cu}(\text { bipy })\left(\mathrm{H}_{2} \mathrm{O}\right) \mathrm{VO}_{34}\right]_{n}$ a coupling more than one order of magnitude smaller than in CuBMB. ${ }^{87}$ Thus, we conclude that our results indicate that the largest exchange couplings in CuBMB are between $\mathrm{Cu}_{1}$ and $\mathrm{Cu}_{3}$ ions, related by inversion operations at distances of $\sim 6.5 \AA$. The DFT calculation confirms these experimental results with $J_{3 a, b}^{\mathrm{c}}>$ $J_{2,4}^{\mathrm{c}}$. Although the relevant point of this work is to learn about the roles of the interactions in the behaviour of the compound, a more detailed quantitative analysis of the superexchange interactions from the structural data remains to be done.

\subsection{Collective spin-behaviour}

Quantum entanglement occurs when pairs or groups of particles interact in such ways that the quantum state of each spin cannot be described independently of the state of the others, but it has to be described for the whole system. Under circumstances described before, this occurs for the $\mathrm{Cu}$ spins in CuBMB as a consequence of the small superexchange couplings, which are evaluated and discussed. EPR measurements allow us to characterize this quantum state of the material, and differentiate it from the uncoupled state, in a case where the small magnitudes of the interactions would make it hard to observe effects in the thermodynamic properties. The phenomenon occurs as a consequence of an avoided crossing ${ }^{32,33}$ of the levels of interacting neighbour coppers, produced by these couplings for a range of orientations of $\boldsymbol{B}_{0}$. It is related to exchange narrowing processes $^{12-14}$ and shows up as a sudden transition produced by the short range of the exchange couplings. In polycrystalline samples where all orientations of $\boldsymbol{B}_{0}$ in the crystal axes occur, the two phases coexist in a range of couplings, as in first order phase transitions, and in a single crystal they can be tuned with the orientation of $\boldsymbol{B}_{0}$. The influence of intermolecular interactions on the magnetic observables has been studied in recent years in connection with interactions that may be unwanted in quantum computing. ${ }^{46,76,88}$ Our approach to the problem uses discoveries made in the beginning of EPR, which are now observed with much greater detail. Our EPR results for single crystal samples at the Q-band are also detected in powder samples where we observe simultaneously the two quantum phases of the spins in the material. The presence of two quantum phases for a system of anisotropic 12 spins can be compared with our previous results for systems of dinuclear copper compounds, ${ }^{41-43}$ where an entangled-spin phase was detected as an unexpected "U-peak" in the powder EPR spectra.

\section{Conclusions}

This work reports the preparation, characterization and structure determination of the new copper compound $\mathrm{Cu}\left(N^{\prime}, N^{\prime}\right.$-dimethyl- $N^{\prime}$ benzoylthiourea)(2,2'-bipyridine)Cl, called CuBMB, and its study with EPR at two microwave frequencies. Its monoclinic crystal structure has two magnetically different (rotated) copper sites with the spin-spin exchange interactions much smaller than the average Zeeman couplings, but comparable with the Zeeman anisotropy of the spin sites. The structural and EPR results allow us to model the electronic properties with a DFT procedure, and characterize the ground state of the $\mathrm{Cu}^{\mathrm{II}}$ ions. The EPR results at room $T$ display a magnetic phase transition between two collective quantum states of the paramagnetic spin system with specific properties and different EPR spectra that can be tuned with the orientation of the magnetic field $\boldsymbol{B}_{0}$ and is characterized by the EPR spectra of a single crystal sample. In one state ("split state") the spins are independent, giving two different EPR peaks; in the other ("merged state") the spins are entangled by the exchange and display a single collective EPR response with line widths showing the exchange narrowing behaviour described by Anderson ${ }^{13}$ and Kubo. ${ }^{14}$ This occurs because the energy levels of each randomly exchange-coupled pair of spins cross at an appropriate applied magnetic field, giving rise to a quantum transition tuned by the field orientation. In powder samples with randomly oriented single crystals one observes a mixture of microcrystals in each phase, with proportions depending on the relative magnitudes of the exchange couplings, and anisotropic Zeeman interactions. Our results display a new view of the classical exchange narrowing problem and are deeply related to present-day advances in problems such as the collective consequences of avoided level crossings produced by exchange couplings, ${ }^{34,35,37}$ Bose-Einstein condensation, ${ }^{38}$ and quantum dynamical phase transitions, ${ }^{44,45}$ where small interactions between magnetic molecules play relevant roles. ${ }^{46,76,88}$ The energy levels of individual pairs of spins which depend on the Zeeman and exchange contributions have avoided crossings that, as in the 
other mentioned cases, produce the observed transitions. The widths of these transitions in angular coordinates displayed in Fig. 5 and 6 depend on the distribution of magnitudes of the exchange interactions within the lattice. The transition is $T$ independent and could in principle be studied in many appropriate paramagnetic crystalline spin arrays. EPR allows studying the characteristics of these phases and following the dynamical properties of the transition. They are also related to transitions of interacting metal-dimeric arrays, ${ }^{39,41-43}$ displaying changes in the EPR spectra which in this case are strongly dependent on $T$ as a consequence of changes in the population of the levels. ${ }^{43}$ We are presently studying other cases of these transitions that, as described by Pastawski, ${ }^{89}$ are ubiquitous in nature, showing up in multiple contexts. For simplicity we did not discuss the effects of the hyperfine couplings provided that they are suppressed by comparatively larger exchange interactions playing a role in the EPR spectra of CuBMB.

\section{Conflicts of interest}

There are no conflicts to declare.

\section{Acknowledgements}

The compound described here was synthesized by BNC as part of her doctoral thesis work. This work was supported by CNPq in Brazil and by the European Social Fund (ESF) under the project CZ.02.2.69/0.0/0.0/18_070/0009469. RC is a member of CONICET, Argentina. We would like to thank Dr Victor Deflon from the Instituto de Química de São Carlos, Universidade de São Paulo, USP, for the crystallographic orientation of the sample used in the EPR measurements.

\section{References}

1 J. Fraxedas, Molecular Organic Materials: From Molecules to Crystalline Solids, Cambridge University Press, Cambridge, 2006.

2 J. Fraxedas, S. V. Malhotra and B. L. V. Prasad, Molecular Materials: Preparation, Characterization, and Applications, CRS Press, Taylor \& Francis, Boca Raton, 2017.

3 V. Ščasnár and J. E. Van Lier, Nucl. Med. Biol., 1993, 20, 257-262.

4 K. Ariga, Q. Ji, M. J. Mcshane, Y. M. Lvov, A. Vinu and J. P. Hill, Chem. Mater., 2012, 24, 728-737.

5 D. Gatteschi, R. Sessoli and J. Villain, Molecular Nanomagnets, Oxford University Press, Oxford, 2006.

6 C. Benelli and D. Gatteschi, Introduction to Molecular Magnetism. From Transition Metals to Lanthanides, Wiley-VCH, Weinheim, 2015.

7 J. Ferrando-Soria, J. Vallejo, M. Castellano, J. Martínez-Lillo, E. Pardo, J. Cano, I. Castro, F. Lloret, R. Ruiz-García and M. Julve, Coord. Chem. Rev., 2017, 339, 17-103.

8 E. Nencki, Ber. Dtsch. Chem. Ges., 1873, 6, 598-600.
9 A. Bielenica, J. Stefańska, K. Stepień, A. Napiórkowska, E. Augustynowicz-Kopeć, G. Sanna, S. Madeddu, S. Boi, G. Giliberti, M. Wrzosek and M. Struga, Eur. J. Med. Chem., 2015, 101, 111-125.

10 A. Bielenica, K. Stepień, A. Napiórkowska, E. AugustynowiczKopeć, S. Krukowski, M. Włodarczyk and M. Struga, Chem. Biol. Drug Des., 2016, 87, 905-917.

11 C. J. Gorter and J. H. Van Vleck, Phys. Rev., 1947, 72, 1128-1129.

12 P. W. Anderson and P. R. Weiss, Rev. Mod. Phys., 1953, 25, 269-276.

13 P. W. Anderson, J. Phys. Soc. Jpn., 1954, 9, 316-339.

14 R. Kubo and K. Tomita, J. Phys. Soc. Jpn., 1954, 9, 888-919.

15 G. E. Pake, Paramagnetic Resonance. An Introductory Monograph, Benjamin, Inc., New York, 1st edn, ch. 7, 1962.

16 A. Abragam, Principles of Nuclear Magnetism, Clarendon Press, Oxford, 1961.

17 M. Yokota and S. Koide, J. Phys. Soc. Jpn., 1954, 9, 953-960.

18 S. K. Hoffmann and L. S. Szczepaniak, J. Magn. Reson., 1983, 52, 182-192.

19 S. K. Hoffmann, J. Goslar and L. S. Szczepaniak, Phys. Rev. B: Condens. Matter Mater. Phys., 1988, 37, 7331-7336.

20 S. K. Hoffmann, W. Hilczer and J. Goslar, Appl. Magn. Reson., 1994, 7, 289-321.

21 M. C. G. Passeggi and R. Calvo, J. Magn. Reson., Ser. A, 1995, 114, 1-11.

22 A. J. Costa-Filho, C. E. Munte, C. Barberato, E. E. Castellano, M. P. D. Mattioli, R. Calvo and O. R. Nascimento, Inorg. Chem., 1999, 38, 4413-4421.

23 A. J. Costa-Filho, O. R. Nascimento, L. Ghivelder and R. Calvo, J. Phys. Chem. B, 2001, 105, 5039-5047.

24 A. J. Costa-Filho, O. R. Nascimento and R. Calvo, J. Phys. Chem. B, 2004, 108, 9549-9555.

25 E. D. Vieira, N. M. C. Casado, G. Facchin, M. H. Torre, A. J. Costa-Filho and R. Calvo, Inorg. Chem., 2006, 45, 2942-2947.

26 R. Calvo, Appl. Magn. Reson., 2007, 31, 271-299.

27 H. Liu and W. Zheng, J. Appl. Phys., 2018, 123, 025105.

28 R. C. Santana, R. O. Cunha, J. F. Carvalho, I. Vencato and R. Calvo, J. Inorg. Biochem., 2005, 99, 415-423.

29 E. F. Chagas, R. E. Rapp, D. E. Rodrigues, N. M. C. Casado and R. Calvo, J. Phys. Chem. B, 2006, 110, 8052-8063.

30 J. A. Weil and J. R. Bolton, Electron Paramagnetic Resonance, Elementary Theory and Practical Applications, Wiley-Interscience, Hoboken NJ, 2nd edn, 2007.

31 R. E. Coffman and G. R. Buettner, J. Phys. Chem., 1979, 83, 2387-2392.

32 C. Zener, Proc. R. Soc. London, Ser. A, 1932, 137, 696-702.

33 L. D. Landau, Phys. Z. Sowjetunion, 1932, 2, 46-51.

34 M. Tachiki and T. Yamada, J. Phys. Soc. Jpn., 1970, 28, 1413-1425.

35 M. Tachiki, T. Yamada and S. Maekawa, J. Phys. Soc. Jpn., 1970, 29, 656-663.

36 M. Tachiki and T. Yamada, Prog. Theor. Phys. Suppl., 1970, 46, 291-309.

37 H. Eleuch and I. Rotter, Fortschr. Phys., 2013, 61, 194-204.

38 V. Zapf, M. Jaime and C. D. Batista, Rev. Mod. Phys., 2014, 86, 563-614. 
39 S. E. Sebastian, P. Tanedo, P. A. Goddard, S.-C. Lee, A. Wilson, S. Kim, S. Cox, R. D. McDonald, S. Hill, N. Harrison, C. D. Batista and I. R. Fisher, Phys. Rev. B: Condens. Matter Mater. Phys., 2006, 74, 180401.

40 L. M. B. Napolitano, O. R. Nascimento, S. Cabaleiro, J. Castro and R. Calvo, Phys. Rev. B: Condens. Matter Mater. Phys., 2008, 77, 214423.

41 M. Perec, R. Baggio, R. P. Sartoris, R. C. Santana, O. Peña and R. Calvo, Inorg. Chem., 2010, 49, 695-703.

42 R. Calvo, J. E. Abud, R. P. Sartoris and R. C. Santana, Phys. Rev. B: Condens. Matter Mater. Phys., 2011, 84, 104433.

43 R. Calvo, V. T. Santana and O. R. Nascimento, Phys. Rev. B: Condens. Matter Mater. Phys., 2017, 96, 064424.

44 S. Sachdev, Quantum Phase Transitions, Cambridge University Press, Cambridge, 2nd edn, 1999.

45 A. A. Zvyagin, Low Temp. Phys., 2016, 42, 971-994.

46 J. Schnack, Phys. Rev. B: Condens. Matter Mater. Phys., 2016, 93, 054421.

47 A. Mohamadou, I. Déchamps-Olivier and J. Barbier, Polyhedron, 1994, 13, 1363-1370.

48 Nonius [or Hooft, R. W. W.], COLLECT, Nonius BV, Delft, The Netherlands, 1998.

49 G. M. Sheldrick, Acta Crystallogr., Sect. A: Found. Crystallogr., 2007, 64, 112-122.

50 P. Coppens, L. Leiserowitz and D. Rabinovich, Acta Crystallogr., Sect. A: Cryst. Phys., Diffr., Theor. Gen. Crystallogr., 1965, 18, 1035-1038.

51 L. J. Farrugia, J. Appl. Crystallogr., 1999, 32, 837-838.

52 C. F. Macrae, I. J. Bruno, J. A. Chisholm, P. R. Edgington, P. McCabe, E. Pidcock, L. Rodriguez-Monge, R. Taylor, J. Van de Streek and P. A. Wood, J. Appl. Crystallogr., 2008, 41, 466-470.

53 S. Stoll and A. Schweiger, J. Magn. Reson., 2006, 178, 42-55.

54 Matlab, The Mathworks Inc.: Natick, MA, USA.

55 F. Neese, Wiley Interdiscip. Rev.: Comput. Mol. Sci., 2017, 8, e1327.

56 S. K. Singh, M. Atanasov and F. Neese, J. Chem. Theory Comput., 2018, 14, 4662-4677.

57 S. I. Levchenkov, I. N. Shcherbakov, L. D. Popov, V. V. Lukov, V. V. Minin, Z. A. Starikova, E. V. Ivannikova, A. A. Tsaturyan and V. A. Kogan, Inorg. Chim. Acta, 2013, 405, 169-175.

58 A. Bencini and D. Gatteschi, J. Am. Chem. Soc., 1986, 108, 5763-5771.

59 A. W. Addison, T. N. Rao, J. Reedijk, J. van Rijn and G. C. Verschoor, J. Chem. Soc., Dalton Trans., 1984, 1349-1356.

60 H. Pérez, R. S. Corrêa, A. M. Plutín, A. Álvarez and Y. Mascarenhas, Acta Crystallogr., Sect. E: Struct. Rep. Online, 2011, 67, o647.

61 N. Gunasekaran, R. Karvembu, S. W. Ng and E. R. T. Tiekink, Acta Crystallogr., Sect. E: Struct. Rep. Online, 2010, 66, o2601.

62 Z. Weiqun, Y. Wen, X. Liqun and C. Xianchen, J. Inorg. Biochem., 2005, 99, 1314-1319.

63 C. Sacht, M. S. Datt, S. Otto and A. Roodt, J. Chem. Soc., Dalton Trans., 2000, 727-733.
64 E. Chalkidou, F. Perdih, I. Turel, D. P. Kessissoglou and

G. Psomas, J. Inorg. Biochem., 2012, 113, 55-65.

65 H. Pérez, B. O’Reilly, A. M. Plutín, R. Martínez, R. Durán, I. G. Collado and Y. P. Mascarenhas, J. Coord. Chem., 2011, 64, 2890-2898.

66 S. Yaseen, M. K. Rauf, S. Zaib, A. Badshah, M. N. Tahir, M. I. Ali, I. ud Din, M. Shahid and J. Iqbal, Inorg. Chim. Acta, 2016, 443, 69-77.

67 K. Nakamoto, Infrared and Raman Spectra of Inorganic and Coordination Compounds, Wiley, New York, 1986.

68 P. W. Anderson, Phys. Rev., 1950, 79, 350-356.

69 P. W. Anderson, Phys. Rev., 1959, 115, 2-13.

70 H. A. Kramers, Physica, 1934, 1, 182-192.

71 J. B. Goodenough, Phys. Rev., 1955, 100, 564-573.

72 J. Kanamori, J. Phys. Chem. Solids, 1959, 10, 87-98.

73 P. J. Hay, J. C. Thibeault and R. Hoffmann, J. Am. Chem. Soc., 1975, 97, 4884-4899.

74 O. Kahn, Molecular Magnetism, Wiley VCH, New York, 1993.

75 W. Wernsdorfer, N. Aliaga-Alcalde, D. N. Hendrickson and G. Christou, Nature, 2002, 416, 406-409.

76 G. A. Timco, S. Carretta, F. Troiani, F. Tuna, R. J. Pritchard, C. A. Muryn, E. J. L. McInnes, A. Ghirri, A. Candini, P. Santini, G. Amoretti, M. Affronte and R. E. P. Winpenny, Nat. Nanotechnol., 2009, 4, 173-178.

77 V. V. Dobrovitski, A. E. Feiguin, D. D. Awschalom and R. Hanson, Phys. Rev. B: Condens. Matter Mater. Phys., 2008, 77, 245212.

78 A. M. Gennaro, P. R. Levstein, C. A. Steren and R. Calvo, Chem. Phys., 1987, 111, 431-438.

79 Y. Servant, J.-C. Bissey and M. Maini, Physica B, 1981, 106, 343-350.

80 R. Calvo and M. A. Mesa, Phys. Rev. B: Condens. Matter Mater. Phys., 1983, 28, 1244-1248.

81 M. Bak and N. C. Nielsen, J. Magn. Reson., 1997, 125, 132-139.

82 A. S. Brill, Transition Metals in Biochemistry, Springer, Heidelberg, 1977.

83 E. I. Solomon, D. E. Heppner, E. M. Johnston, J. W. Ginsbach, J. Cirera, M. Qayyum, M. T. Kieber-Emmons, C. H. Kjaergaard, R. G. Hadt and L. Tian, Chem. Rev., 2014, 114, 3659-3853.

84 R. Taylor and O. Kennard, J. Am. Chem. Soc., 1982, 104, 5063-5070.

85 G. A. Jeffrey, An Introduction to Hydrogen Bonding, Oxford University Press, Oxford, 1997.

86 R. Zhao and R.-Q. Zhang, Phys. Chem. Chem. Phys., 2016, 18, 25452-25457.

87 D. Venegas-Yazigi, K. A. Brown, A. Vega, R. Calvo, C. Aliaga, R. C. Santana, R. Cardoso-Gil, R. Kniep, W. Schnelle and E. Spodine, Inorg. Chem., 2011, 50, 11461-11471.

88 A. Ardavan, A. M. Bowen, A. Fernandez, A. J. Fielding, D. Kaminski, F. Moro, C. A. Muryn, M. D. Wise, A. Ruggi, E. J. McInnes, K. Severin, G. A. Timco, C. R. Timmel, F. Tuna, G. F. Whitehead and R. E. Winpenny, npj Quantum Information, 2015, 1, 15012.

89 H. M. Pastawski, Physica B, 2007, 398, 278-286. 